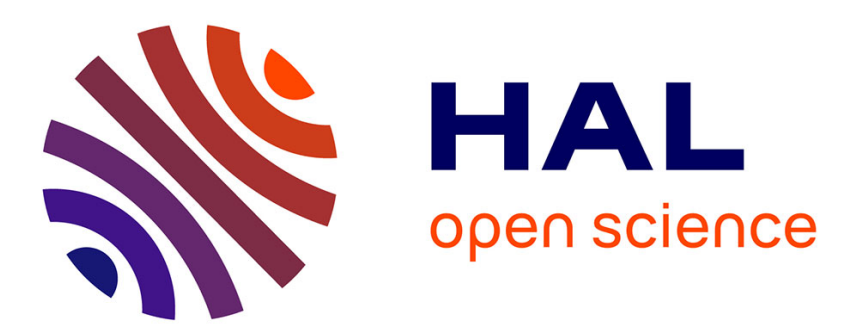

\title{
Effects of the preferential segregation of droplets on evaporation and turbulent mixing
}

Julien Reveillon, François-Xavier Demoulin

\section{To cite this version:}

Julien Reveillon, François-Xavier Demoulin. Effects of the preferential segregation of droplets on evaporation and turbulent mixing. Journal of Fluid Mechanics, 2007, 583, pp.273-302. 10.1017/S0022112007006180 . hal-00474853

\section{HAL Id: hal-00474853 \\ https://hal.science/hal-00474853}

Submitted on 21 Apr 2010

HAL is a multi-disciplinary open access archive for the deposit and dissemination of scientific research documents, whether they are published or not. The documents may come from teaching and research institutions in France or abroad, or from public or private research centers.
L'archive ouverte pluridisciplinaire HAL, est destinée au dépôt et à la diffusion de documents scientifiques de niveau recherche, publiés ou non, émanant des établissements d'enseignement et de recherche français ou étrangers, des laboratoires publics ou privés. 


\title{
Effects of the preferential segregation of
}

\section{droplets on evaporation and turbulent}

\author{
mixing \\ By JULIEN REVEILLON \\ AND FRANCOIS-XAVIER DEMOULIN \\ University of Rouen \\ CORIA UMR-CNRS 6614 \\ Avenue de l'Université \\ 76801 Saint Etienne du Rouvray \\ FRANCE \\ corresponding author: reveillon@coria.fr
}

(Received 15 November 2006) 


\section{Abstract}

Droplet segregation in isotropic homogeneous turbulence is analyzed using a spectral DNS solver to describe the evolution of the turbulent carrier phase, whose characteristic properties remain statistically stationary thanks to a semi-deterministic forcing scheme. Lagrangian dilute spray modeling is employed to describe the discrete phase evolution. The liquid density is distributed on the Eulerian mesh to analyze the evolution of the spray and its spatial distribution. This gives results in accordance with classical methods as far as droplet segregation is concerned. Moreover, it allows a deeper analysis of the spray evolution. In particular, droplet segregation and vapour mass fraction may be jointly analyzed. First, droplet segregation phenomena are studied through the analysis of the formation and the geometry of the droplet clusters. Then, the effects of segregation on spray evaporation are investigated from both the dispersed and carrier phase points of view. At equilibrium, droplet dynamics leads to different segregation levels that are associated with characteristic Stokes numbers. It appears that the evaporation process evolves following three different stages in time: single droplet mode in the early stages, cluster mode in the intermediate stages and a gaseous mode in the late stages. Segregation levels strongly affect the evolution of the mean vapour mixture fraction during the second stage, while the corresponding standard deviation is affected longer, up to the third stage in our simulations. However, from the evolution of the integral scale and the shape of the energy spectrum, it appears that turbulent mixing erases the segregation effects apart from the first evaporation stage when the droplet segregation rules the vapour distribution. 


\section{Introduction}

In industrial systems dedicated to transport or energy transformation, a combustion chamber is generally fuelled by a spray of evaporating liquid droplets. Depending on the chamber's geometry, the spray injection conditions and the mixing phenomena, various combustion regimes and flame structures may be observed, starting from premixed flames up to diffusion flames. However, partially premixed combustion is generally observed because of the triple interactions between spray, turbulence and combustion. The modeling of such chambers, involving the combustion of a two-phase flow or of a gaseous mixture released by the evaporation of a spray, is of primary importance to improve the output of the device considered and to predict pollutant formation while still maintaining a reasonable development cost.

One of the main input parameters of any non-premixed turbulent combustion model is the mixture fraction variable (denoted $Z$ in this paper), which characterizes locally the mixing between the evaporated fuel and the gaseous oxidizer. Indeed, in Reynolds averaged (RANS) modeling and according to the selected combustion model, the mean mixture fraction, its variance and its dissipation rate allow the generation of presumed probability density functions (PDF, Borghi (1988)) or the localisation in flamelet libraries (Peters (1986)). These parameters are also the basis of conditional moment closure methods (Klimenko \& Bilger (1999)). It is possible to note that within the framework of large eddy simulations (LES), analogous data have to be determined at a subgrid level.

Much work has been dedicated to predicting the mixture fraction evolution in purely gaseous flows where $Z$ is an inert scalar: the evolution of its averaged value $\bar{Z}$ is classically established through an advection/diffusion equation although determining its variance $\overline{Z^{\prime 2}}$ and dissipation $\chi_{Z}$ is less straightforward; see for instance the work of Newman et al. (1982), Mantel \& Borghi (1994) and references therein. The difficulties are now well 
understood and many closures exist. However, when two phase flows are considered, the mixture fraction is no longer an inert scalar. Indeed, the appearance, in the gas phase, of mass source terms due to the evaporation of the liquid phase modifies deeply the mixture fraction field. According to the local droplet density, $Z$ fluctuations appear during the turbulent mixing and the local rise of mixture fraction gradients affects the dissipation rate. The formalism of these new source terms and their effects on the gas phase have been described in some recent studies: Reveillon et al. (1998); Reveillon \& Vervisch (2000); Demoulin \& Borghi (2002); Colin \& Benkenida (2003) where new closures have been suggested. These models evaluate the new source terms appearing in the transport equations for mixture fraction mean and variance. These models also take into account the evaporation effects on the dissipation level and the mixing delay.

However, while interesting data about the effects of these mass source terms have been gathered, there is a lack of information concerning their spatial distribution. Indeed, prior to the determination of the gas phase turbulent mixing, dispersion of evaporating droplets is a major phenomena that directly affects the evolution of the mixture fraction . Although the dispersion of particles embedded in turbulent flows is one of the major research fields in the two-phase flow community, no direct link has been made between the droplet turbulent dispersion, the mass source term distribution and the mixture fraction evolution in a generic configuration.

The objective of this paper is to describe the simultaneous effects of turbulence on spray dispersion and mixture fraction evolution. A direct numerical simulation (DNS) solver has been coupled with a Lagrangian model to describe the evolution of an evaporating spray in a turbulent flow. Thus, the main characteristics of the mixture fraction field (fluctuation intensity levels and characteristic length scales) are estimated directly 
with respect to the local properties of the turbulence (rms, length scales) and the spray parameters (Stokes number, liquid equivalence ratio).

As sketched in figure 1, multiple interactions between the turbulent flow, the spray dispersion and the vapour micro-mixing may be defined. To reduce the number of varying parameters, a synthetic configuration very close to the one used by Eswaran \& Pope (1988), for their reference study dedicated to the turbulent mixing of purely gaseous flow, has been extended to two-phase flow simulations. Thus, a forced isotropic homogeneous turbulence, with statistically stationary properties, is used as the carrier phase. A recently developed forcing scheme ( Guichard et al. (2004)) allowing stable mean properties of the turbulence to be obtained (energy, dissipation, integral length scale) has been introduced in a spectral formulation describing the evolution of the gas phase.

Over the last decade, DNS has been widely used in a large range of applications. It was first introduced for inert flow simulations (Orzag \& Patterson (1972); Rogallo (1981); Lee et al. (1991)) and has been extended to reactive flows in order to study non-premixed, partially premixed and premixed turbulent combustion of purely gaseous fluids (Givi (1989); Poinsot et al. (1996); Vervisch \& Poinsot (1998); Poinsot \& Veynante (2001); Pantano et al. (2003)). DNS has been extended to two-phase flows since the pioneering work of Riley \& G.S. Patterson (1974). Most of the first numerical studies were dedicated to solid particle dispersion (see for instance: Samimy \& Lele (1991); Squires \& Eaton (1991); Elgobashi \& Truesdell (1992); Wang \& Maxey (1993); Ling et al. (1998)). Recently, Mashayek et al. (1997) conducted one of the first DNS with evaporating droplets in turbulent flows. In this study they neglected the effect of the spray on the carrier phase (one-way coupling) and they assumed an incompressible flow. Since then, DNS of two-phase flows have been extended to incorporate two-way coupling 
effects and to deal with spray evaporation and combustion phenomena (Mashayek (1998);

Miller \& Bellan (1999, 2000); Reveillon \& Vervisch (2005)).

In this work, various physical analyses are obtained from three-dimensional simulations following a two-stage procedure. First, the turbulent dispersion of an initially randomly dispersed spray is considered with non-evaporating particles. According to their Stokes number and the properties of the turbulence, the formation of clusters of particles is analyzed (formation delay, characteristic cluster size). In addition to the criterion proposed by Fessler et al. (1994) to characterize the length scale of the clusters, a new analysis is proposed to link the spray segregation with the future mixture fraction field. Indeed, once the particles have reached a dynamical equilibrium with the surrounding turbulent flow, evaporation phenomena are considered through the analysis of the mixture fraction evolution. Our goal is to observe the impact of the preferential segregation of the droplets on the mixture fraction field.

In this work, a one-way coupling has been considered. Indeed, when a two-way coupling is used (Mashayek (1998); Reveillon \& Vervisch (2000, 2005)), the impact of the spray on the carrier phase modifies the turbulence characteristics and it becomes difficult to isolate the various interactions between dispersion, evaporation and mixing. Of course, the flow evolution considered is more realistic, but in the framework of this paper we decided to work on synthetic configurations where the main varying parameter is the droplet's Stokes number, which leads to various local segregation and mixture fraction fields. Thus, it will be possible to determine the sole impact of the spray segregation on the mixture fraction fields that are undergoing a similar turbulent mixing. Moreover, it would not be logical to associate a realistic drag source term with an artificial turbulence forcing term in the momentum equation of the gas phase.

In the subsequent sections, the DNS configurations and the control parameters are 
detailed together with a description of the governing equations and the various hypotheses that have been used. Then, a brief description of the statistical tools is offered before the various results are analyzed in two stages: a preliminary description of the solid particle dispersion and a study of the effects of the evaporation of the droplets on the mixture fraction field.

\section{Governing equations and modeling}

Various sets of governing equations are coupled together to carry out the computations. Because of the forcing procedure restrictions, the gas phase velocity is evaluated in spectral space. The evolution of the mixture is described in physical space so that it can be easily coupled with the Lagrangian description of the evaporating spray.

\subsection{Gas velocity and turbulence forcing}

The gaseous carrier phase is an isotropic homogeneous turbulence that is resolved in a cubic domain with periodic boundary conditions. To maintain the major properties (energy, dissipation rate, integral scale) of the spectral turbulence near to constant values, a controlled amount of energy must be transferred into the spectral simulation through a forcing procedure.

There are various ways to achieve the forcing of isotropic homogeneous turbulence in a spectral DNS. First, it is possible to freeze the magnitude of the largest structures of the spectral velocity field Siggia \& Patterson (1978); Sirovich (1991). But the stabilisation of the flow parameters requires many eddy turn-over times and the results are statistically dependent of the large anisotropic structures. Another solution is to use stochastic schemes Eswaran \& Pope (1988), where energy is added randomly in the low wave-number modes. These schemes appear to be efficient and statistically independent. But some turbulence properties fluctuated too widely around their prescribed values . A 
third way is to use a deterministic forcing scheme which forces the lower-wave number modes with a controlled amount of energy. One of the most effective studies in this domain has been carried out by Overholt \& Pope (1998). Considering the forcing methods of Kerr (1981) and Siggia \& Patterson (1978), the Overholt and Pope deterministic forcing scheme forces the simulated spectrum towards a model spectrum by using a timeand wave number-dependent coefficient. Recently Guichard et al. (2004) extended this method and proposed a Fully Controlled Deterministic Forcing Scheme (FC-DFS) with a more efficient convergence rate towards the model spectrum; this reduced the fluctuations of the prescribed properties drastically. The FC-DFS scheme has been used in this work.

Turbulence is forced by adding a linear source term to the balance equation for the velocity field $\widehat{u}$ in wave number space:

$$
\frac{\partial \widehat{u}}{\partial t}=\widehat{a}+\frac{f_{\kappa}}{\tau_{f}} \widehat{u}
$$

where $\widehat{a}$ represents the classical Navier-Stokes contributions for an incompressible flow. The forcing function $f_{\kappa}\left(\kappa_{F}, \Delta \kappa_{F}\right)$ is real and depends on both time $t$ and wave number magnitude $\kappa$. The principle of the model is to relax the simulated spectrum $E_{s}$ towards a model spectrum $E_{m}$ only for a given range of low wave numbers $\left(\kappa<\kappa_{F}\right)$. This method has been detailed and tested Guichard et al. (2004). However, all the simulations are based on this forcing procedure. Thus a quick description is given in the following.

With the new source term (eq. 1.1), the time evolution of the simulated energy spectrum $E_{s}$ may be written:

$$
\frac{d E_{s}}{d t}=C_{\kappa}+2 \frac{f_{\kappa}}{\tau_{f}} E_{s}
$$

where $C_{\kappa}$ represents the usual energy contributions without any source term. The objective is to attract the simulated spectrum $E_{s}$ towards a model spectrum $E_{m}$. This may be 
done by inserting directly an attraction parameter in the equation of the time evolution of the energy spectrum:

$$
\frac{d E_{s}}{d t}=C_{\kappa}+F_{\kappa} \frac{\alpha_{f}(t) E_{m}(\kappa)-E_{s}(\kappa, t)}{\tau_{f}}
$$

where $F_{\kappa}$ is a filter function varying from zero to unity:

$$
F_{\kappa}=\frac{1-\exp \left(-\frac{\left(\kappa-\kappa_{f}\right)^{2}}{\Delta \kappa^{2}}\right)}{1-\exp \left(-\frac{\kappa_{f}^{2}}{\Delta \kappa^{2}}\right)} H\left(\kappa_{f}-\kappa\right),
$$

which determines the amplitude of the forcing for every wave number. $\mathrm{H}(\mathrm{x})$ is the Heavyside function defined by $H(x)=1$ if $x>0$ and $H(x)=0$ if $x<0$. The filter function allows a smooth transition between the relaxed and the non-relaxed zones. $\tau_{f}$ is a characteristic relaxation delay of the simulated spectrum $E_{s}$ towards a model spectrum $E_{m}$. To keep the system stable, $\tau_{f}$ must be smaller than the smallest time scale of the flow Overholt \& Pope (1998). The Kolmogorov time scale

$$
\tau_{\kappa}=\sqrt{\frac{\nu}{\langle\varepsilon\rangle}}
$$

has thus been used with the following definition : $\tau_{f}=C_{f} \tau_{\kappa}$, where $C_{f}$ is a constant coefficient (set to 0.5 in our simulations), $\nu$ is the fluid viscosity and $\langle\varepsilon\rangle$ is the current mean dissipation, determined over the whole spectral domain.

Note that, to maintain the simulated integral length scale in the vicinity of the modeled one while letting the inertial range evolve freely, the cut-off $\left(\kappa_{f}\right)$ has to be selected at the 'beginning' of the inertial range. By identifying equation 1.2 with equation 1.3 , the forcing function $f_{\kappa}$ may be directly defined by the following expression:

$$
E_{s}(\kappa, t) f_{\kappa}=\frac{1}{2} F_{\kappa}\left(\alpha_{f}(t) E_{m}(\kappa)-E_{s}(\kappa, t)\right)
$$

The coefficient $\alpha_{f}$ controls the evolution of the mean turbulent energy $\langle k\rangle$ in the 
spectral domain. This is achieved by assuming the following arbitrary evolution:

$$
\frac{d\langle k\rangle}{d t}=\frac{k_{\infty}-\langle k\rangle}{\tau_{f}}
$$

For sake of simplicity, it is assumed that the target value $\left(k_{\infty}\right)$ corresponds to the energy of the model spectrum:

$$
k_{\infty}=\int_{\kappa} E_{m}(\kappa) d \kappa
$$

To determine $\alpha_{f}$, the equation 1.7 is compared with the theoretical definition of the mean kinetic energy, which is obtained by integrating the equation 1.2 :

$$
\frac{d\langle k\rangle}{d t}=-\langle\varepsilon\rangle+\frac{2}{\tau_{f}} \int_{\kappa} f_{\kappa} E_{s}(\kappa, t) d \kappa
$$

By integrating over $\kappa$ the equation 1.6 and by introducing the relations 1.7 and 1.9 , an expression for $\alpha_{f}$ may be derived:

$$
\alpha_{f}(t)=\frac{k_{\infty}-\langle k\rangle+\tau_{f}\langle\varepsilon\rangle+\int_{\kappa} F_{\kappa} E_{s}(\kappa, t) d \kappa}{\int_{\kappa} F_{\kappa} E_{m}(\kappa) d \kappa} .
$$

The forcing function $f_{k}$ is obtained with the following equation:

$$
f_{\kappa}=\frac{F_{\kappa}}{2 E_{s}(\kappa, t)}\left(\alpha_{f}(t) E_{m}(\kappa)-E_{s}(\kappa, t)\right)
$$

This relation allows for attracting the simulated spectrum towards the shape of a model spectrum and to control the mean kinetic energy.

\subsection{Scalar transport}

Because of the constraints of the forcing procedure, a spectral solver is used to describe the evolution of the turbulent velocity of the flow. A one-way momentum coupling determines the droplet motion. A two-way coupling could be used, although there would be no point in associating it with the artificial forcing term of the turbulence. Moreover, this one-way coupling allows us to compare the preferential segregation of various sprays undergoing exactly the same turbulent flow. 
On the other hand, to study the evolution of the mixture fraction released by the droplet evaporation, a two-way coupling is considered from the mass exchange point of view. The time evolution of species mass fractions is thus considered in a physical space to simplify their coupling with the dispersed phase.

The following classical relation applies for the vapour mass fraction:

$$
\frac{\partial Y_{F}}{\partial t}+\frac{\partial u_{i} Y_{F}}{\partial x_{i}}=D \frac{\partial^{2} Y_{F}}{\partial x_{i}^{2}}+\frac{1}{\rho} \dot{\omega}_{v}
$$

where $D$ is the species diffusion coefficient and $\dot{\omega}_{v}$ the mass source term due to the evaporation of the dispersed liquid phase. A similar equation without source term is considered for the carrying gas mass fraction: $Y_{O}$. The velocity components $u_{i}$ are obtained from an inverse Fourier transform of the spectral velocity field: $u_{i}=T \cdot F \cdot{ }^{-1}(\widehat{u})$.

These equations are solved with the third order Runge-Kutta procedure already used for the spectral space. Similar sub time-steps are applied for both spectral and physical solvers. A sixth order Pade scheme from Lele (1992) allows the spatial derivatives to be determined. As for the spectral velocity field, periodic boundary conditions have been used.

\subsection{Liquid phase dispersion}

A discrete Lagrangian approach is adopted to follow the spray evolution within the gaseous oxidizer. It is now discussed.

By denoting $\mathbf{v}_{k}$ and $\mathbf{x}_{k}$ the velocity and position vectors of every droplet $k$, the following relations:

$$
\begin{aligned}
\frac{d \mathbf{v}_{k}}{d t} & =\frac{1}{\beta_{k}^{(v)}}\left(\mathbf{u}\left(\mathbf{x}_{k}, t\right)-\mathbf{v}_{k}\right) \\
\frac{d \mathbf{x}_{k}}{d t} & =\mathbf{v}_{k}
\end{aligned}
$$

are used to track the evolutions of the droplets throughout the computational domain. 


\section{J. Réveillon AND F.X. Demoulin}

The vector $\mathbf{u}$ represents the gas velocity at the droplet position $\mathbf{x}_{k}$. The right hand side term of equation 1.13 stands for a drag force applied to the droplet where $\beta_{k}^{(v)}$ is a kinetic relaxation time:

$$
\beta_{k}^{(v)}=\frac{a_{k}^{2}}{a_{0}^{2}} \frac{\tau_{p}}{C_{k}}
$$

$a_{k}$ is the diameter of the droplet $k$ and $a_{0}$ is the initial diameter of any droplet of the initially monodispersed spray. The characteristic kinetic time $\tau_{p}$ is defined by the relation

$$
\tau_{p}=\frac{\rho_{d} a_{0}^{2}}{18 \mu}
$$

and a corrective coefficient $C_{k}=1+R e_{k}^{2 / 3} / 6$ (Crowe et al. (1998)) is introduced to allow for the variation of the drag factor according to the value of the droplet's Reynolds number $R e_{k}=\rho\left|\mathbf{u}\left(\mathbf{x}_{k}, t\right)-\mathbf{v}_{k}\right| a_{k} / \mu$.

The evaporation of every droplet in the flow accounts for the fuel mass fraction at the droplet surface $Y_{k}^{s}$ and the local vapour level at the droplet position, $Y_{F}\left(\mathbf{x}_{k}\right)$. Saturation conditions mainly depend on the properties of the flow at the droplet's surface: temperature, pressure. In our simplified configuration, a constant saturation level such as $Y_{k}^{s}=Y^{s}$ is considered.

The following relation for the surface evolution of every " $k$ " droplet may be written:

$$
\frac{d a_{k}^{2}}{d t}=-\frac{a_{k}^{2}}{\beta_{k}^{(a)}}
$$

A classical model (d-square law) is to consider $\beta_{k}^{(a)}=$ cte. It leads to a linear relation between droplet surface and time. However, in our simulation, saturation is accounted for and the characteristic relaxation time is defined by:

$$
\beta_{k}^{(a)}=\frac{S_{c}}{4 S h_{c}} \frac{\rho_{d}}{\mu} \frac{a_{k}^{2}}{\ln \left(\frac{1-Y_{F}}{1-Y^{s}}\right)},
$$

where $S c=0.7$ is the Schmidt number and $S h_{c}$ the convective Sherwood number, which is equal to 2 in a quiescent atmosphere; however, a correction must be applied in a 
convective environment. In this context, the empirical expression of Faeth and Fendell (Kuo (1986)) has been used:

$$
\left(S h_{c}\right)_{k}=2+\frac{0.55 R e_{k} S c}{\left(1.232+R e_{k} S c^{4 / 3}\right)^{1 / 2}}
$$

The mean evaporation delay is controlled by selecting an appropriate value for the saturation level $Y^{s}$. Usually, the evaporation delay is defined as the time necessary for a droplet to be vaporized, assuming that the mass fraction of vapour far from the droplet is constant. If this mass fraction of vapour is $Y_{F}=0$, the vaporization time is:

$$
\tau_{v}=\frac{S c}{4 S h_{c}} \frac{\rho_{d}}{\mu} \frac{a_{0}^{2}}{\ln \left(\frac{1}{1-Y^{s}}\right)} .
$$

However, when saturation laws apply, it is possible to compute a dynamic characteristic evaporation delay $\tau_{v}^{s}$ of a droplet in a quiescent atmosphere that takes into account the varying level of fuel vapour around the liquid. Figure 2 shows the evolution of the ratio $\tau_{v}^{s} / \tau_{v}$ versus the saturation level $Y^{s}$ for a given initial mass fraction of liquid $Y_{0}^{l}$. Such an effect can be important for the evaporation of liquid fuel since generally the saturation value is low since the temperature of the atomized liquid is far from boiling temperature.

\subsection{Coupling term}

The term $\dot{w}_{v}$ affects the mixture fraction evolution owing to a distribution of the Lagrangian mass on the Eulerian grid. Every droplet has mass source terms to be dispatched over the Eulerian nodes and the organization of an accurate projection of those Lagrangian sources to the Eulerian mesh is not straightforward. In a real spray flow, this distribution is not instantaneous and further assumptions are needed. In our simulations, each Lagrangian source is distributed over Eulerian nodes directly surrounding the droplet. A finite volume approach is applied considering the intersection of the elementary volume centered around the droplet and the elementary volume centered around 
each node (Fig. 3). This induces a numerical dispersion that remains weak because of the small size of the DNS grid and the small turbulent Reynolds number (Reveillon \& Vervisch (2000)).

For every Eulerian node, a control volume $\mathcal{V}$ is defined by the mid-distance between the neighbour nodes. Because an isotropic Cartesian grid has been used $\left(\Delta=x_{i+1}-x_{i}=\right.$ $\Delta_{x}=\Delta_{y}=\Delta_{z}$ ), the volume $\mathcal{V}$ is defined by $\mathcal{V}=\Delta^{3}$. The mass source term applied to any Eulerian node $n$ is denoted $\dot{w}_{v}^{(n)}$ :

$$
\dot{w}_{v}^{(n)}=\frac{1}{\mathcal{V}} \sum_{k}-\alpha_{k}^{(n)} \frac{d m_{k}}{d t},
$$

where $\sum_{k}$ is the sum over all the droplets affecting the node $n . \alpha_{k}^{(n)}$ is the distribution coefficient of the $k$ droplet source term on the node $n$. Considering all the nodes affected by the droplet $k$, it is necessary to have $\sum_{n} \alpha_{k}^{(n)}=1$ to conserve mass, momentum and energy during the Lagrangian/Eulerian coupling. In fact, $\alpha_{k}^{(n)}$ is the portion of the control volume of the node $n$ intersecting the control volume of the droplet $k$ (Fig. 3):

$$
\alpha_{k}^{(n)}=\frac{1}{\mathcal{V}} \prod_{i=1}^{3}\left(\Delta-\left|x_{i}^{(n)}-x_{k i}\right|\right),
$$

where $x_{i}^{(n)}$ and $x_{k i}$ are the coordinates along the $i_{t h}$ direction of the node $n$ and the droplet $k$ respectively.

The mass of the considered $k$ droplet in the neighbourhood of the node is defined by $m_{k}=\rho_{d} \pi a_{k}^{3} / 6$ and, using equations 1.17 and 1.21 , one may write

$$
\dot{w}_{v}^{(n)}=\rho_{d} \frac{\pi}{4} \frac{1}{\mathcal{V}} \sum_{k} \alpha_{k}^{(n)} a_{k}^{3} / \beta_{k}^{(a)}
$$

\section{Configurations and statistical considerations}

The following three-stage procedure has been employed to analyse all the interactions between the turbulent flow and the dispersed phase. Vorticity contours and dispersing 
particles have been plotted in Fig. 4 to illustrate both Eulerian and Lagrangian resolutions.

Stage 1: statistically stationary turbulence

In this preliminary stage, the turbulent gaseous phase evolves alone in a $129^{3}$ Cartesian grid until its statistical properties reach a steady state thanks to the forcing procedure that keeps the mean kinetic energy $k=3.375 \mathrm{~m}^{2} / \mathrm{s}^{2}$ at the prescribed level (fig. 5-(a)-top). The mean dissipation rate $\varepsilon$ is not specifically a prescribed parameter but it must reach a steady state $\left(\varepsilon=2.7510^{4} \mathrm{~m}^{2} / \mathrm{s}^{3}\right)$, as shown in figure 5-(a)-bottom. The energy spectrum allows us to determine a reference wave number $\kappa_{0}$ corresponding to turbulence scales that contain most of the kinetic energy. The corresponding physical length $l_{0}=2 \pi / \kappa_{0} /$ is an integral scale of the flow that has been used as a reference parameter in this work along with its spectral counterpart $\kappa_{0}$. In all the simulations, $\kappa_{0}=7 \Delta \kappa . \Delta \kappa$ is the spectral grid size defined by $\Delta \kappa=2 \pi / L$ where $L$ is the cubical domain dimension equal to $3 \mathrm{~mm}$. The maximum energy length $l_{0}$ is thus equal to $l_{0}=L / 7$ and remains constant as shown in figure 5-(a)-middle. The velocity root mean square $u^{\prime}=1.5 \mathrm{~m} / \mathrm{s}$, is used as reference parameter along with the eddy turn-over time $\tau_{0}=l_{0} / u^{\prime}$ and the characteristic time of the velocity fluctuations $\tau_{\kappa}$ of the smallest structures $\left(\eta \approx 1.810^{-5} \mathrm{~m}\right)$. The turbulent Reynolds number of the simulation is $R e_{l_{0}}=43$ and the parameters of the filter function, as defined by Guichard et al. (2004), are $\kappa_{F}=9 \Delta \kappa$ and $\Delta \kappa_{F}=3 \Delta \kappa$.

Stage 2: spray dynamical equilibrium

Several eddy turn-over times after the turbulent flow reaches its stationary state, $N_{d} \approx$ 2150000 mono-dispersed non-evaporating particles are randomly embedded throughout the computational domain with a zero initial velocity. The drag force, described above in 


\section{J. Réveillon AND F.X. Demoulin}

equation 1.13 , sets particles in motion. Then, the spray reaches a dynamical equilibrium with the turbulence (Fig. 4-(b)). It corresponds to a stationary slip velocity standard deviation. Droplet dispersion is usually characterized by Stokes number $S t=\tau_{p} / \tau_{\kappa}$ (Wang \& Maxey (1993)), which indicates the ability of droplets to capture local variations of the carrier phase velocity. Turbulence properties being fixed, simulations have been carried out by modifying the $\tau_{p}$ parameter. By doing so, 12 various Stokes numbers varying between 0.025 and 11 have been considered.

To characterize droplet dispersion and preferential concentration, a density $\xi(\mathbf{x}, t)$, describing the local mass of liquid per unit of volume has been defined. To make the spectral analysis of physical properties easier, the Eulerian computational grid has been used and $\xi(\mathbf{x}, t)$ is determined by considering the droplets accumulated around each node. A mean reference mass density is defined by $\bar{\xi}_{0}=m_{d} N_{d} / L^{3}$ where $m_{d}$ is the initial mass of each droplet (monodispersed spray). The constant parameter $\bar{\xi}_{0}$ is used to normalize the evolution of $\xi(\mathbf{x}, t)$. Thus, in a non-evaporating mode, $\xi(\mathbf{x}, t) / \bar{\xi}_{0}$, which may also be considered as a number density, has a constant unitary mean $\bar{\xi}(t)=L^{-3} \int \xi(\mathbf{x}, t) d \mathbf{x}=\bar{\xi}_{0}$ over the whole domain and its standard deviation $\xi^{\prime}(t)=L^{-3}\left(\int(\xi(\mathbf{x}, t)-\bar{\xi}(t))^{2} d \mathbf{x}\right)^{(1 / 2)}$, normalized by $\bar{\xi}_{0}$ in the following, identifies the droplet segregation level. Note that, in the following, $\overline{()}$ stands for the mean over the Eulerian grid whereas $(\tilde{)}$ is the mean over the Lagrangian particles or droplets.

$\underline{\text { Stage 3: liquid phase evaporation and micro-mixing }}$

When the droplets are evaporating, $\bar{\xi} / \bar{\xi}_{0}$ is bounded between 1 and $0 . \bar{\xi} / \bar{\xi}_{0}$ provides information about the evaporation state whereas $\xi^{\prime} / \bar{\xi}$ characterizes the droplet segregation. Using the parameter $\xi$ to characterize the spray evolution and preferential segregation is not the usual choice when compared with other work dedicated to the analysis of prefer- 
ential segregation in sprays, but it allows well established results to be retrieved (Fessler et al. (1994)). The method was thus chosen in accordance with the main objective of this paper, which is to analyze the correlations between the droplet concentration and the micro-mixing of the gaseous fuel released by the liquid evaporation. Using a Eulerian droplet density allows a direct comparison with the Eulerian mixture fraction field $Z$.

In the last part of this work, once the particles have reached a dynamical equilibrium with the surrounding turbulence, they are allowed to evaporate according to a specific characteristic evaporation delay $\tau_{v}$ prescribed by setting the saturation level as described above. Thus, for a given initial non-evaporating configuration, various simulations with different evaporation delays will be carried out and analyzed.

To describe the mixing between the gaseous fuel and the oxidizer, the usual definition of the mixture fraction $Z$ is chosen (Linán \& Williams (1993); Poinsot \& Veynante (2001)) leading to $Z=Y_{F}$ in an inert flow. A passive scalar is defined as $\varphi=s_{r} Y_{F}-Y_{O}$ (Linán \& Williams (1993)), where the stoichiometric mass ratio is $s_{r}=11$ when considering the single step reaction $\mathrm{C}_{7} \mathrm{H}_{16}+11 \mathrm{O}_{2} \rightarrow 7 \mathrm{CO}_{2}+8 \mathrm{H}_{2} \mathrm{O}$. Normalizing $\varphi$ yields to the mixture fraction $Z=\left(\Phi_{o}\left(Y_{F} / Y_{F, o}\right)-\left(Y_{O} / Y_{O, o}\right)+1\right) /\left(\Phi_{o}+1\right)$, with $Y_{F, o}=1, Y_{O, o}=0.23$ and $\Phi_{o}=s_{r} Y_{F, o} / Y_{O, o}$. Note that in the case of spray evaporation, $Z$ cannot reach unity, but a local maximum level depending on saturation conditions. Consequently, a normalization of $Z$ is introduced by using the saturation limit $Z_{s} . Z / Z_{s}$ is thus bounded between 0 and 1 and it is of practical interest for analyzing correlations between the evaporating spray and the turbulent mixing.

\section{Results and discussion}

Although homogeneous turbulence is a straightforward configuration, the addition of an evaporating dispersed phase allows us to be at the midpoint of many poorly under- 
stood interactions between turbulence, spray, mixing and combustion. As described in the introduction, for the sake of clarity this paper focuses on the correlations between the mixture fraction field and the preferential droplet segregation. The analysis of flame ignition and propagation or model closures will be the purpose of future work. In the first part, the equilibrium regimes of non-evaporating droplets are depicted before evaporation takes place. Then evaporation is considered using both a liquid and a gas analysis. In particular, the evolution of the mixture fraction PDF is studied. Finally, the topologies of the liquid and vapour fields are investigated thanks to a spectral analysis.

\subsection{Liquid dispersion}

The equilibrium of the spray with its surrounding carrier phase is detected through the Lagrangian statistics of the slip velocity $\mathbf{w}_{k}=\left(\mathbf{u}\left(\mathbf{x}_{k}, t\right)-\mathbf{v}_{k}\right)$, where $\mathbf{u}$ is the gas velocity at the droplet location and $\mathbf{v}$ is the droplet velocity. Because of the homogeneous nature of the turbulence and the dispersion, the mean value of the slip velocity defined by $\tilde{\mathbf{w}}=$

$N_{d}^{-1} \sum_{k=1}^{N_{d}} \mathbf{w}_{k}$ remains equal to zero. However, as shown in figure 5-(b), the slip velocity root mean square $w^{\prime \prime}=\sqrt{w_{i} w_{i}}$ evolves toward a stationary value $w_{\infty}^{\prime \prime}$ corresponding to the equilibrium of the spray with the surrounding gas. Because particles are initially randomly distributed in the computational domain with a zero velocity, $w^{\prime \prime} / u^{\prime}$ is unitary at time $t=0$. Indeed, $u^{\prime}$ stands for the r.m.s. of the gas velocity, sampled with the initial field of particles. Then, as described previously, the drag forces set the particles in motion and, depending on the Stokes numbers, $w^{\prime \prime}$ reaches a steady state in less than $0.1 \tau_{0}$ (Fig. 5 -(d)) for a small Stokes number $(S t=0.35)$ whereas $1.6 \tau_{0}$ is needed for the largest value of the Stokes number $(S t=11)$. This is an important point because, in more complex configurations like combustion chambers, even though evaporation and ignition delays are short, droplets are most certainly already in dynamical equilibrium when evaporation takes place. The final mean stationary value of the slip velocity standard 
deviation: $w_{\infty}^{\prime \prime}$ is in plotted figure 5-(c) with respect to the droplet's Stokes number. This value is zero when the droplets are small enough to follow all the velocity fluctuations of the flow, and it increases regularly to reach asymptotically the value one, corresponding to droplets that are too heavy to move $(S t=\infty)$.

To study the preferential concentration of discrete particles in turbulent flows, several approaches exist: see for instance Squires \& Eaton (1991); Wang \& Maxey (1993); Fessler et al. (1994); Simonin et al. (1993); Aliseda et al. (2002). If a statistically homogenously distributed spray is randomly injected, i.e. if there is no preferential segregation, the distribution of the number of particles per control volume (CV) of a given size must follow a binomial distribution, which may be approximated by a Poisson distribution in our configurations. Hence, the study of the preferential concentration is usually based (Fessler et al. (1994)) on the difference between the actual segregated distribution and the Poisson distribution. It is characterized by

$$
\Sigma=(\sigma-\sqrt{\lambda}) / \lambda
$$

where $\lambda$ is the average number of particles per cell whereas $\sigma$ and $\sqrt{\lambda}$ are the standard deviations of the particle distribution and the Poisson distribution, respectively. For a given Lagrangian distribution of the particles, $\Sigma$ depends strongly on the size of the CV considered. However, according to Fessler et al. (1994), the length scale corresponding to the characteristic cluster size is equal to $\Delta_{\Sigma \max }$, which is the size of the CV when $\Sigma$ reaches a maximum value.

In the case considered in this work, the particles are liquid droplets of fuel that are evaporated to prepare the reactive mixture. Preferential concentrations of particles are potentially important when describing induced heterogeneities that could appear in the mixture fraction field. Following such considerations, another parameter, more represen- 
tative of the evaporation and turbulent mixing processes, has been considered to describe preferential segregation effects.

Droplet dispersion and preferential segregation have been analyzed from a Eulerian point of view thanks to the local Eulerian liquid density $\xi(\mathbf{x}, t)$. Instantaneous fields of $\xi$ have been plotted in figure 6 for three Stokes numbers $(S t=0.17, S t=1.05$ and $S t=5.6)$ along with the corresponding vorticity field. These four fields have been captured at exactly the same time after droplet dispersion has reached a stationary value $\left(t>t_{\infty}\right)$. Even without any quantitative analysis, it is possible to notice the dramatic impact of the particles' inertia on their dispersion properties. Indeed, even with a small Stokes number, particles tend to leave the vortex cores and segregate in weak vorticity areas. This phenomenon may be seen in figures 6-(b) and 6-(c) where $\xi$ is represented for $S t=0.17$ and $S t=1.05$, respectively. This last case shows a normalized liquid density $\xi / \bar{\xi}_{0}$ ranking between 0 (no droplets) and 5 (five times the mean density). As will be shown later, density fluctuations reach a maximum when $S t=1$. When $S t=0.17$, segregation is already clearly visible (maximum deviation : 2.5) although there are more intermediates density areas (Fig. 6-(b)). When the $S t=1$ limit is 'broken', the droplet distribution tends to be totally different than for $S t \leq 1$, see Fig. 6-(d) . Indeed, kinetic times become large enough for the droplets to cross high vorticity areas, leading to a less segregated spray (maximum deviation: 2.5). This result is confirmed in figure 7 where the mean liquid density conditioned by the local vorticity level has been plotted for various Stokes numbers. For highly segregated sprays $(S t=1.05)$, high vorticity areas are almost empty, with an average value of $\bar{\xi} / \bar{\xi}_{0}$ equal to 0.2 , whereas when vorticity tends to $0, \bar{\xi} / \bar{\xi}_{0}$ converges towards 2 . The figure 7 is in accordance with classical results such as the work of Squires \& Eaton (1991). They found a maximum of correlation between the number of particle and the vorticity level for a Stokes number equal to 0.15 . However, they used 
a Stokes number based on the integral time scale of the turbulence. By swapping it by the Kolmogorov time scale, the peak of correlation occurs for a unitary Stokes number. Indeed the Kolmogorov scaling is necessary to characterize segregation effect as outlined by Wang \& Maxey (1993). When $S t=0.17$ and $S t=5.6$ the correlation between low vorticity areas and high droplet density clusters is less pronounced. However, because of the ballistic nature of the heavy droplets $(S t=5.6)$ the high vorticity areas are still densely populated. This confirms the qualitative result of figure $6-(d)$.

To evaluate the preferential segregation of the droplets embedded in the turbulent flow, $\xi_{\infty}^{\prime}$ has been plotted in figure 8-(a). This parameter is the standard deviation of the field $\xi$ when droplets are in dynamical equilibrium with the carrier phase $\left(t>t_{\infty}\right)$. Starting from $S t=0.025$ with $\xi_{\infty}^{\prime} / \bar{\xi}_{0}=0.55$, a maximum segregation $\xi_{\infty}^{\prime} / \bar{\xi}_{0}=1.4$ is observed for a unitary Stokes number before a progressive decay. This parameter informs us of the characteristic liquid density level in clusters that have been formed by the turbulent structures.

The variable $\xi$ is not classically used to capture the properties of dispersed particles, therefore, the results have been compared in figure 8-(b) with the maximum of $\Sigma$, which is the deviation from the Poisson distribution as defined in equation 3.1. Both $\Sigma$ and $\xi_{\infty}^{\prime}$ being standard deviations of different variables, quantitative comparisons are not relevant in this case and the data have been normalized by their maximum value, when $S t=1$. The evolution of the deviation $\xi_{\infty}^{\prime}$ with respect to the Stokes number is very similar to that of the classical parameter $\Sigma$, although $\xi_{\infty}^{\prime}$ is slightly higher when $S t>3$. However, both curves reach a maximum value when $S t=1$. Wang \& Maxey (1993) or Fessler et al. (1994) have shown also a similar dependence of the segregation intensity on the Stokes number. This confirms that the parameter $\xi$ defined in this work is relevant to characterize segregation. 
Similarly, it is possible to determine, with various methods, the characteristic size of the clouds (or clusters) of particles. In this paper, we used the information obtained from the energy spectrum $E_{\xi}(\kappa)$ (Fig. 9) of the variable $\xi(x, t)$ when $t>t_{\infty}$, and then we extracted $l_{\xi}=2 \pi / \kappa_{\xi}$ where $\kappa_{\xi}$ is the position, in spectral space, of the most energetic level. Plotting the $l_{\xi}$ dependence with the Stokes number in figure 10 demonstrates the large-scale effects of the turbulence on the spray. The cluster sizes computed from $E_{\xi}$ have been compared (Fig. 10) with the ones obtained thanks to the classical Lagrangian way of Fessler et al. (1994). In this last approach, the cluster size denoted $\Delta_{\Sigma \max }$ is the width of the CV when $\Sigma$ reaches a maximum value. Both $l_{\xi}$ and $\Delta_{\Sigma \max }$ show a similar evolution with a minimum size for $S t=1$. In their experiment Aliseda et al. (2002) compared the characteristic cluster size $\Delta_{\Sigma \max }$ to the length scale found using a method proposed by Wang \& Maxey (1993). In this last approach, the change between the actual distribution to the binomial distribution is measured as the square of the difference of probabilities given by the two distribution summed over all possible values. Aliseda et al found that both methods lead to the same characteristic length scale for the cluster. Thus, using the Eulerian $\xi$ parameter is in accordance with the classical Lagrangian approach to the analysis of preferential segregation. Notice that for the measurement of the characteristic cluster length scale, Aliseda et al. (2002) used all the droplet class sizes. Thus it is not possible to determine the Stokes number dependency of the clusters characteristic length scale. The two methods they used to find the cluster length scale agree globally when considering all the droplet sizes. It is still possible that some difference may appear for specific values of Stokes as we found when comparing $\Delta_{\Sigma \max }$ to $\xi$.

Fessler et al. (1994) conducted experiments with various sets of particles corresponding to Stokes numbers ranging from 1.7 up to 130. Their study does not extend to Stokes numbers smaller than unity. It appears that $\Delta_{\Sigma \max }$ is dependent on the Stokes number, 
starting with a value of the order of the Kolmogorov length scale, it increases as the Stokes number increases. For Stokes number greater than unity, the parameter $\xi$ allow this trend to be recovered. In our DNS, when $S t<1$, the evolution of the cluster size obtained with both $\Sigma$ and $\xi$ is similar although the length scales are different (Fig. 10). However, using $\xi$ as reference parameter offers a new range of possible analyses developed below.

From a phenomenological point of view, the cluster size evolution results from the competition between three physical phenomena: the ejection of the droplets from the vortex cores by the turbulence, the turbulent micro-mixing (prevalent when $S t<1$ ) and the ballistic effects (prevalent when $S t>1$ ). Indeed, droplets tend to be ejected from the turbulent structures to form clusters concentrated in low vorticity areas. However, for droplets with a small Stokes number, turbulent micro-mixing counteracts the segregation process and 'diffuse' clouds are obtained (as seen in figure 6-(b)): the lighter the droplets, the more effective the mixing and the larger the characteristic size of the cluster. When $S t=1$, an optimal segregation is obtained because micro-mixing is weak and the droplets are not heavy enough to leave low vorticity areas where they are trapped. However, as soon as inertia is prevalent $(S t>1)$ the particles are able to cross turbulent structures no matter what their vorticity is and the characteristic size of the clusters increases again. In figure 10, it is clear that both $l_{\xi}$ and $\Delta_{\Sigma \max }$ capture this natural dependence on the Stokes number.

To conclude on the non-evaporating dispersion aspect, our results show that preferential segregation in spray cannot be characterized using only the mean segregation parameter $\xi_{\infty}^{\prime}$ and the mean density $\bar{\xi}$. Indeed, for two sprays with the same mean density and whose Stokes numbers are 0.17 and 5.6, a similar mean segregation level equal to 0.85 is found in figure 8-(a). However, the corresponding topologies of spray density are 
distinct, as may be seen in figures 6-(b) and 6-(d) where $\xi$ is plotted for $S t=0.17$ and $S t=5.6$, respectively. This dramatic difference is confirmed in figure 10 by the characteristic sizes of the clusters of particles equal to 0.29 and 0.65 when normalised by the characteristic length scale of the most energetic turbulent structures $l_{o}$. Consequently, for two fields with identical first moments of $\xi$, different mixture fraction topologies can be obtained from droplet evaporation and different combustion regimes might be observed if the ignition delay is short compared to the turbulent mixing time. Thus, in addition to $\bar{\xi}$ and $\xi_{\infty}^{\prime}$, a third parameter, which has to depend on the droplet's Stokes number, is necessary to describe accurately the preferential segregation of the spray and the subsequent mixture fraction field.

\subsection{Evaporation and turbulent mixing}

Once dynamical equilibrium is reached between the turbulent gaseous flow and the dispersed phase, evaporation is activated. Mixing between the fuel vapour and the gaseous oxidizer is characterized by the mixture fraction $Z$. It is bounded between zero, when there is no vapour, and a limit value $Z_{s}$, which is the saturation level. Three cases are chosen with three different initial Stokes numbers. They are recapitulated in table 1: cases B have a Stokes number close to unity when segregation is maximum, cases A and C correspond to low and high Stokes numbers, respectively. As detailed previously, their segregation parameter $\xi^{\prime}$ is the same, although the characteristic cluster size and the droplet dynamics are very different. Various characteristic evaporation delays $\tau_{v}$ have been considered : (1) $\tau_{v}=0.5 \tau_{0}$, (2) $1 \tau_{0}$, (3) $2 \tau_{0}$. Configuration names are summarised in table 1 as well.

The evolution of $\bar{Z} / Z_{s}$ is plotted in figure 11-(a) for cases A1, B1 and C1. It is clear from this figure that the most segregated case (B1, unitary Stokes number) takes the longest time to be evaporated although a first order estimation (obtained thanks to the tangent 
of the curve at $t / \tau_{0}=0$ ) would give the same results for the three cases. Note that both configurations $\mathrm{A} 1$ and $\mathrm{C} 1$ start with exactly the same evolution of the mean mixture fraction. Indeed, the initial droplet dispersion level being the same in both cases, the evaporation rate is similar at first and only a small discrepancy, which will be explained later in the article, appears when $t / \tau_{0}=0.2$. No matter what the Stokes number of the droplets is, the mean mixture fraction evolution depends only on the initial segregation level of the particles. If the segregation level is small enough to avoid the creation of saturated pockets of fuel vapour, whatever the dynamics (or the Stokes number) of the droplets is, the evolution of $\bar{Z}$ may be easily estimated by a classical d-square law. On the other hand, if the segregation level and mass of liquid fuel are high enough for the pocket of vapour to reach the saturation limit, the evolution of $\bar{Z}$ is affected. Indeed, clusters containing a large number density of droplets are quickly surrounded by a high level of vapour concentration. Consequently, the gaseous fuel diffusion flux from the droplet surface to the surrounding gas is reduced because of the saturation that strongly decreases the evaporation rate for each droplet embedded in any cluster. Evaporation may even stop if the vapour concentration reaches locally the saturation limit. Consequently, the droplets need more time to evaporate. Some of them, generally the heaviest ones, may be ejected from the cluster by turbulent motion to reach areas where vapour concentration is low enough for them to evaporate. On the other hand, the smallest ones may be trapped in the cluster. In that case, the driving mechanism of the evaporation is linked to the global diffusive flux of vapour from the saturated cluster towards areas with low vapour concentration.

A very different behaviour may be observed in figure 11-(b) where the evolution of the standard deviation of the mixture fraction : $Z^{\prime}$ has been plotted for cases A1, B1, C1. From a general point of view, the global shape of the curves is the same: starting 
from $Z^{\prime} / Z_{s}=0$ when evaporation starts, the curves reach a maximum value long before the characteristic evaporation delay (when $t / \tau_{v} \approx 0.2$ ). It is here that the dissipation effects on the mixture fraction fluctuations become greater than the effects of the evaporation. Consequently, the mixture fraction fluctuations decrease continuously. Details of the competition between evaporation and dissipation may be found in Reveillon \& Vervisch (2000).

A more detailed analysis shows different local evolutions of the mixture fraction. First, the most segregated spray generates the most fluctuating mixture fraction field (case B1, $S t=1.05)$. Indeed, droplets are accumulated in small clusters and when evaporation starts, high levels of mixture fraction are obtained in small control volumes leading to a strong deviation $Z^{\prime}$. Another important point to notice is the evolution of the deviation $Z^{\prime}$ of the case $\mathrm{C} 1$ that initially evolves like case $\mathrm{A} 1$ because of the initial similar droplet segregation level. However, it very quickly exceeds (more than 30\%) the maximum value of $Z_{A 1}^{\prime}$ before joining, almost exactly, the curve $Z_{B 1}^{\prime}$ corresponding to the highly segregated case. This behaviour, totally different from that observed previously in the evolution of $\bar{Z}$, is due to the decrease of the mean Stokes number of the spray that leads to a very quick segregation of the evaporating droplets of case C1. On the other hand, there is no change in segregation for case A1 $(S t=0.17)$ as the Stokes number becomes smaller and smaller.

This interpretation is confirmed in figure 12 where the evolution of the droplet segregation parameter $\xi^{\prime}$ and the droplets' mean Stokes number have been plotted for cases A3, B3, C3 $\left(\tau_{v}=2 \tau_{0}\right)$, which shows this behaviour more clearly. The evolution of spray segregation represented by the standard deviation of the droplet density $\xi^{\prime}$ is shown in figure 12-(a). The general trend of cases B and A is a decrease of the segregation because of the diminution of the corresponding Stokes number that may be seen in figure 12-(b). 
The turbulent micro-mixing becomes more and more effective leading to a less segregated spray. When the Stokes number is initially greater than unity (cases C) the segregation first increases before following the general decay. The initial elevation of the segregation level corresponds to an evolution of the droplet dynamics to a level that is more efficient (unitary Stokes number) to create clusters and, therefore, to increase $\xi^{\prime}$ momentarily. This modification of the evaporating droplet dynamics has a direct impact on the mixture fraction evolution as seen in figure 11-(b). It also explains the small discrepancy between cases $\mathrm{A}$ and $\mathrm{C}$ in the evolution of $\bar{Z}$ (Fig. 11-(a)) underlined previously. Indeed the temporary formation of clusters in case C leads to slightly more saturated clouds of fuel vapour . Consequently, it delays the evaporation process.

The evolution of the mean droplet surface divided by its initial value is shown in figure 13 for various Stokes numbers and various evaporation delays. Additional curves, represented by circles, stand for the mean droplet surface evolution that would be obtained if there were no preferential segregation (homogeneous droplet distribution). This curve may be obtained analytically thanks to equations 1.17 and 1.18 where $Y_{F}$ is replaced by the mean mass fraction of fuel increasing in the domain. This model has been used to determine the characteristic evaporation delay $\tau_{v}$. Figure 13-(a) demonstrates the dramatic effect of the segregation of the dispersed phase on the evaporation process. Two distinct stages may be observed. First, when evaporation starts, far from the saturation limit, the decrease in the mean droplet surface area calculated from the DNS is similar to the results obtained with the analytical model that neglects the segregation phenomena. Then, depending on the Stokes number (i.e. on the droplet segregation level), a second evaporating stage may be observed with a slower rate. This behaviour is confirmed by figure 13-(b) where the surface evolution has been plotted for a given Stokes number (St $=0.17)$ and various evaporation delays. 


\section{J. Réveillon AND F.X. Demoulin}

The evaporation scenario is shown in figure 14 and may be summarised as follow:

(1) Initially the evaporation is very quick since no vapour concentration prevents it.

(2) Soon the cluster that contains a high density of droplets reaches a vapour concentration close to saturation. Consequently, the evaporation rate of the droplets inside the cluster is strongly diminished, if not stopped. Then, two evolutions are possible : (3a) The lightest droplets are not able to leave the saturated area because of their lack of inertia. Their evaporation rate is thus strongly related to the diffusion rate of the pocket of vapour toward the fresh gases. In this case, all the droplets inside a cluster could be considered as a single entity, which mimics the evaporation law for a single droplet. (3b) Turbulent motion contributes to dispersing the heaviest droplets. Then, they may reach non-saturated areas to finish their evaporation process.

\subsection{Mixture fraction PDF}

There are various ways to describe the statistics of the vapor mass fraction. In the preceding section, evolutions of the first two moments of $Z$ have been plotted in figure 11 to characterize the direct impact of the droplet segregation on the corresponding mixture fraction fluctuations. A thorough statistical analysis can be carried out by detailing the transformation of $P\left(Z / Z_{s}\right)$, the probability density function (PDF) of the mixture fraction variable $Z$ normalised by the saturation value $Z_{s}$. By doing so, the phase space is bounded between 0 and 1, facilitating the analysis. General time evolutions of $Z / Z_{s}$ are plotted in figure 15 for the three reference Stokes numbers: $S t=0.17, S t=1.05$ and $S t=5.6$. Starting from a Dirac function (not represented) at $Z / Z_{s}=0$ when evaporation begins, the PDF evolves, with a bell shaped curve, towards another Dirac function corresponding to the final mean mixture fraction in the volume.

More details may be found in figure 16 where snapshots of the PDF shapes have been plotted for three different times. The left figure shows the three PDFs when $t=0.025 \tau_{0}$. 
Although the mean mixture fraction is small, it is clear that the curves corresponding to $S t=0.17$ and $S t=5.6$ are similar for short times. This is a remarkable property. Indeed, we already knew, thanks to figure 8-(a), that the segregation parameter $\xi^{\prime}$, and therefore, the mixture fraction variance $Z^{\prime}$, were similar in the early stages of the evaporation for cases $S t=0.17$ and $S t=5.6$. However, the corresponding cluster sizes (Fig. 10) were different, possibly leading to different mixture fraction topologies. It appears in figure 16left that the PDF shape is not strongly affected by the various cluster sizes in the early stages of the evaporation process. Thus, not only the first two moments are similar but also the moments greater than two. However, numerous models are based on the first two moments of the mixture fraction variable and it is interesting to note that those models, often deduced from the analysis of the mixing of a purely gaseous flow, may be used for two phase flows if the evaporation source terms are correctly accounted for.

Two other plots in figure 16 confirm the general evolution noticed in figure 11-(b) when the Stokes number of the heaviest droplets reaches the optimal segregation level corresponding to $S t=1$. Consequently the PDF of case $C_{1}$ relaxes toward the PDF of case $B_{1}$. At an intermediate stage, (center plot of figure 16), both $S t=0.17$ and $S t=5.6 \mathrm{PDF}$ are still centered around a similar mean value but their shapes are no longer alike. The difference between both shapes is considerable and it is clear that combustion models based on such a PDF would give contrasted results. The last point to be noticed in figure 16-center, is the fact that, apart from case $S t=0.17$, the PDFs are not symmetrical around the mean. Indeed, cases $S t=0.17$ and $S t=5.6$ correspond to dispersing droplets that can be found outside the pockets of vapour generated around droplet clusters because of turbulent-micromixing or ballistic effects. This peculiar behaviour leads to an increased probability of finding small mixture fraction levels. At this stage it is difficult to determine 
if combustion would be strongly affected by this result. However, if it is the case, it would be necessary to take into account the lack in symmetry of the PDF in the models.

\subsection{Spatial distribution of the mixture fraction field}

The last part of this work is dedicated to the study of the parallel evolution of both the spray segregation and the mixture fraction field during the evaporation process. As detailed above in this article, among the various ways to describe this field we selected a spectral formulation that may be used for both dispersed and gaseous phases.

Stationary spectra of the liquid density fluctuations $\xi^{\prime}$ have been plotted in figure 17 for the three standard Stokes numbers $S t=0.17, S t=1.05$ and $S t=5.6$, along with that of the turbulent velocity field. Discussions of the energetic levels of these spectra and their evolution have been conducted above through the analysis of the standard deviation of $\xi$ and $Z$, and the corresponding spectra have been plotted in figure 9. Thus, to focus on the spectral and physical topologies of both fields, $E_{\xi}$ and $E_{Z}$ have been normalized by the variance of the field analyzed so that the integral of all the spectra presented is unitary. Moreover, the wave number direction $\kappa$ is normalized by $k_{0}$, the reference wave number of the turbulent velocity field corresponding to the frequency of the most energetic structures.

Before evaporation starts, the normalized shapes of $E_{\xi}$ spectra (Fig. 17) corresponding to configurations $S t=0.17$ and $S t=1.05$ are very similar, with a peak of energy centered in a frequency range three to four times greater than that of the turbulence. When $S t=5.6$, the low frequency spectrum shape is different from the other two and very close to the shape of the velocity energy. Indeed, the heavy droplets are mainly influenced by the large turbulent structures of the flow. On the other hand, the three spectra present high energy rates for the high frequency corresponding to the sampling of isolated droplets or small clouds of droplets. 
The time evolution of normalized spectra $E_{Z}$ (left) and $E_{\xi}$ (right) is plotted for two different evaporation delays: $\tau_{v}=\tau_{0} / 2$ in figure 18 and $\tau_{v}=2 \tau_{0}$ in figure 19 for three different Stokes numbers $S t=0.17, S t=1.05$ and $S t=5.6$ (from top to bottom). In each figure, several spectra have been drawn: curves with square symbols represent the initial spectra corresponding to the very beginning of the evaporation. Then the spectra (thin black curves) evolve towards the spectrum with circle symbols corresponding to the characteristic evaporation delay. Spectra evolving from this moment are represented with gray lines.

Several general conclusions may be drawn from these figures. First of all, the time evolutions of all $E_{Z}$ spectra clearly follow an identical path whatever the droplet's Stokes number and evaporation delay. When evaporation starts, the initial normalized mixture fraction spectra $E_{Z}$ have exactly the same shape as the corresponding $E_{\xi}$ spectra. However, diffusion and turbulent micro-mixing affect the evolution of $E_{Z}$ whereas the modification of the droplet dynamics and evaporation transforms $E_{\xi}$. Small structures of mixture fraction due to isolated droplets or small clusters disappear very quickly and there is a shift of the peak of energy towards the low frequency range. In other words, the characteristic size of the vapour pockets becomes progressively larger. It is a natural behaviour due to the disappearance of inhomogeneities because of turbulent mixing of the gas phase. Another contribution may be the stochastic behaviour of the droplets or small clusters leading to a vapour wake larger than the effective cluster size. During this process an inertial range appears on the mixture fraction spectra (Figs. 18 and 19, left). In a logarithmic representation, this range seems to converge towards the $k^{-5 / 3}$ limit that has been represented on the figures. Particle density spectra are not concerned by the apparition of an inertial range. Indeed it is a characteristic behaviour of turbulent gaseous mixing. As it has been described by Mydlarski \& Warhaft (1998), this inertial 
range may be observed for all Reynolds number unlike the velocity spectrum that needs high values of $R e$. From this point of view, the spray evaporation has a weak effect on the shape of the scalar spectra. It appears that the time necessary to develop an inertial range does not depend on the evaporation delay. Indeed, the effects of turbulence on the shapes of $E_{Z}$ spectra are prevalent compared to the impact of the evaporation of the remaining droplets. Thus, in the configurations considered, the evaporation source terms mainly affect the energy level of the spectrum but not really its shape, except in the early stages of the evaporation process where there exists a Stokes dependence of the length scale (similar when $S t=0.17$ and $S t=1.05$ and twice as large when $S t=5.6$ ). In that short period of time, the mixture fraction spectrum matches that of the clusters of droplets. This is confirmed by figures 20-(a) and 21-(a) where the characteristic length scales $l_{Z}$ of the mixture fraction follow almost exactly the same evolution whatever the evaporation delays or the Stokes number. Combustion is thus susceptible to be affected directly by the size of the clusters only if the ignition delays are short; otherwise, the main driving mechanisms will be the turbulent micro-mixing and the mean evaporation rate. Of course, it must not be forgotten that clusters of droplets may affect combustion processes in other ways, in particular through the momentum (drag forces) or temperature (droplet evaporation) coupling.

The evolution of $E_{\xi}$ spectra depends strongly on the droplet's Stokes number and the evaporation delay. As seen in figures 18 and 19, various behaviors may be considered. If $\tau_{v}=\tau_{0} / 2$ and $t<\tau_{v}$ (Figs. 18), the spectrum shapes remain almost stationary. Then, in a second phase, there is a shift of the maximum of the spectra towards the high frequency modes implying a decrease of the characteristic size of the cluster of droplets because of the evaporation. This shift is more or less pronounced depending on the initial Stokes number of the droplet. This behaviour is shown graphically in figure 20-(b) where $l_{Z}$ 
has been plotted for $S t=0.17, S t=1.05$ and $S t=5.6$ for $\tau_{v}=\tau_{0} / 2$. The cluster size remains almost stationary during the main evaporation process. But as soon as droplets begin to disappear, cluster sizes decay naturally. It is possible to note the quick evolution of the $S t=5.6$ case.

$E_{\xi}$ spectra of case $\tau_{v}=2 \tau_{0}$ (Figs. 19) have a very different evolution. Indeed, instead of keeping the same global shape and shifting towards high frequencies, a bimodal shape appears during evaporation. Indeed, while the high frequency range always sustains a high energy level, the energy of the intermediate frequency range begins to decay strongly while the low frequency energy increases. As soon as the characteristic evaporation delay is reached, low frequency energy decays and the few remaining droplets concentrate the density fluctuations in the high frequency modes as might be expected. Considering these spectrum shapes, it appears that, for a while, two extreme characteristic length scales may be defined. This leads to a sudden modification of $l_{\xi}$ (Fig. 21-(a)) because each spectrum peak swaps from high frequency to low frequency. The specific appearance of a double length scale is confirmed in figure 22-(a) where thin-fingered structures (small length scale ) are emerging from clusters of droplets (large length scale). On the contrary, in figure 22-(b) only one prevalent structure may be detected as confirmed by figures 18 .

\section{Conclusion}

Statistically stationary turbulence is used as carrier phase to study spray dispersion and evaporation thanks to a spectral DNS solver where a semi-deterministic forcing scheme has been implemented. The evolution of the dispersed liquid phase is modelled using a classical Lagrangian description. A one-way coupling is applied between the Eulerian and Lagrangian solvers except for the description of the vapour mass fraction evolution, which uses a two-way coupling. Consequently, turbulent flow characteristics 
remain constant while the dispersion of various sprays is studied. The sole effect of the droplet dynamics has thus been isolated and characterized for various sprays by prescribing Stokes numbers ranging between 0.025 and 11 . The liquid density $\xi$ is introduced to characterize droplet segregation properties. This quantity offers results similar to classical Lagrangian methods (Fessler et al. (1994)) but deeper analysis is possible, in spectral space for example.

To begin with, the dispersion of non-evaporating droplets is considered. It shows the formation of clusters of particles whose size and dynamics are strongly dependent on the Stokes number considered. This segregation effect is the result of three competing phenomena: the natural ejection of the droplets from the high vorticity areas, the turbulent micro-mixing that affects the smallest droplets, and the ballistic effects, associated to the heaviest ones. In the second stage, droplet evaporation has been considered. Then, the consequences of preferential segregation on the whole evaporation process are studied in detail from both the gaseous and liquid points of view. Several conclusions have been drawn from this study and have been summarized in table 2 .

Overall, the presence of droplet clusters due to preferential segregation is important for global variables such as the mean vapour mass fraction $\bar{Z}$ and its standard deviation $Z^{\prime}$, which are the key parameters of any combustion model. Hence presence and properties of clusters must be accounted for. However, it appears that knowing the mean liquid density and its standard deviation is not sufficient to characterize the spray dispersion. An additional parameter, directly linked to droplet dynamics, such as the Stokes number, is thus necessary. Independently of the initial Stokes number, three stages can be defined for droplet evaporation:

(a) single droplet evaporation mode: far from saturation, there is no influence of clus- 
ters and an almost d-square law whose rate depends on isolated droplet characteristics is observed.

(b) cluster evaporation mode: droplets are embedded in vapour pocket close to saturation. The evaporation rate strongly diminishes. It depends on the ability of the droplets to leave the saturated cloud (ballistic effect, high Stokes number) and on the gaseous diffusion flux from vapour saturated pockets towards low vapour concentration areas.

(c) gaseous mode: the droplets have vanished or their number is no longer large enough to influence the gas phase evolution. The classical turbulent mixing controls the vapour evolution.

During droplet evaporation, mixture fraction field was scrutinized as well. First, an analysis of the evolution of the PDF of the mixture fraction has been carried out for three reference Stokes numbers. For very short times, the PDF shapes are not significantly different, and this corresponds to the single droplet evaporation mode. However, as soon as vapour pockets reach the saturation level, PDFs are strongly affected. If ignition should occur at this moment, very different flames would be observed according to the droplet dynamics. Then, when the evaporation rates become negligible (gaseous mode) turbulent micro-mixing prevails and the mixture fraction PDFs relax towards a classical Gaussian shape. Eventually, a joint spectral analysis of both the mixture fraction and liquid density spectra showed the strong impact of the cluster formation on the mixture fraction field, especially in the early stages of the evaporation process. However, turbulent mixing and gaseous diffusion prevail very quickly and vapor spectra relax towards a similar shape independently of their initial Stokes number. Note that the global energy level remains strongly affected by the liquid density spatial distribution. On the contrary, the liquid density spectra are strongly influenced by the droplet dynamics and differences still persist up to the end of the evaporation process. However, because the evaporation rate 
becomes negligible, the liquid density distribution no longer affects the mixture fraction field.

\section{Acknowledgment}

CPU time was provided by IDRIS-CNRS (Institut du Développement et des Ressources en Informatique Scientifique). The authors would like to thank Dilys Moscato for a thorough reading and the made suggestions, which have improved the paper.

\section{REFERENCES}

Aliseda, A., Cartellier, A., Hainaux, F. \& Lasheras, J. 2002 Effect of preferential concentration on the settling velocity of heavy particles in homogeneous isotropic turbulence. J. Fluid Mech. 468, 77-105.

Borghi, R. 1988 Turbulent combustion modelling. Prog. Energy Combust. Sci. 14, 245-292.

Colin, O. \& Benkenida, A. 2003 A new scalar fluctuation model to predict mixing in evaporating two-phase flows. Combustion and Flame 134, 207-227.

Crowe, C., Sommerfeld, M. \& Tsuji, Y., ed. 1998 Multiphase flows with droplets and particles. CRC Press.

Demoulin, F. \& Borghi, R. 2002 Modeling of turbulent spray combustion with application to diesel like experiment. Combustion and Flame 129, 281-293.

Elgobashi, S. \& Truesdell, G. 1992 Direct numerical simulation of particle dispersion in a decaying isotropic turbulence. J. Fluid Mech. 242, 655-700.

Eswaran, V. \& Pope, S. 1988 Direct numerical simulations of the turbulent mixing of the passive scalar. Phys. Fluids 31 (3), 506-520.

Fessler, J., Kulick, J. \& EAton, J. 1994 Preferential concentration of heavy particles in turbulent channel flow. Phys. Fluids (6), 3742-3749.

Givi, P. 1989 Model free simulations of turbulent reactive flows. Prog. Energy Combust. Sci. 15, $1-107$.

Guichard, L., Reveillon, J. \& Hauguel, R. 2004 Direct numerical simulation of statistically 
stationary one- and two-phase turbulent combustion: a turbulent injection procedure. Flow, turbulence and combustion 73, 133-167.

KERR, R. M. 1981 Theoritical investigation of passive scalar such as temperature in isotropic turbulence. PhD thesis, Cornell University.

Klimenko, A. \& Bilger, R. 1999 Conditional moment closure for turbulent combustion. Prog Energy Combust Sci 25, 595-687.

Kuo, K., ed. 1986 Principles of combustion. John Wiley and sons.

Lee, S., Lele, K. \& Moin, P. 1991 Numerical simulations of spatially evolving compressible turbulence. Center for Turbulence Research, Annual Research Briefs, Stanford (126).

LELE, S. K. 1992 Compact finite difference schemes with spectral like resolution. J. Comput. Phys. (103), 16-42.

LinÁn, A. \& Williams, F. 1993 Fundamentals aspects of combustion. Oxford University Press.

Ling, W., Chung, J., Troutt, T. \& Crowe, C. 1998 Direct numerical simulation of a threedimensional temporal mixing layer with particle dispersion. Journal of fluids mechanics (358), 61-85.

Mantel, T. \& Borghi, R. 1994 A new model of premixed wrinkled flame propagation based on a scalar dissipation equation. Combustion and Flame 96, 443-457.

MAshayeK, F. 1998 Direct numerical simulations of evaporating droplet dispersion in forced low mach number turbulence. Int. j. heat mass transfer 41 (17), 2601-2617.

Mashayek, F., Jaberi, F., Miller, R. \& Givi, P. 1997 Dispersion and polydispersity of droplets in stationary isotropic turbulence. Int. J. Multiphase Flow 23 (2), 337-355.

Miller, R. \& Bellan, J. 1999 Direct numerical simulation of a confined three-dimensional gas mixing layer with one evaporating hydrocarbon-droplet-laden stream. Journal of fluids mechanics 384, 293-338.

Miller, R. \& Bellan, J. 2000 Direct numerical simulation and subgrid analysis of a transitional droplet laden mixing layer. Phys. Fluid 12 (3), 650-671.

Mydlarski, L. \& WARhaft, Z. 1998 Passive scalar statistics in high-pclet-number grid turbulence. Journal of fluids mechanics 358, 135-175. 
Newman, G., Launder, B. \& Lumley, J. 1982 Modeling the behavior of homogeneous scalar turbulence. Journal of fluids mechanics 111, 217-232.

Orzag, S. A. \& Patterson, G. S. 1972 Numerical simulation of three-dimensional homogeneous isotropic turbulence. Phys. Rev. Lett. 28, 76.

Overholt, M. R. \& Pope, S. B. 1998 A deterministic forcing scheme for direct numerical simulation of turbulence. Computer and Fluid 27, 11-28.

Pantano, C., Sarkar, S. \& Williams, F. 2003 Mixing of a conserved scalar in a turbulent reacting shear layer. J. Fluid Mech 481, 291-328.

Peters, N. 1986 Laminar flamelet concepts in turbulent combustion. In Proceedings of the 21rst Symposium (International) on combustion (ed. P. The combustion institute), pp. $1231-1250$.

Poinsot, T., Candel, S. \& Trouvé, A. 1996 Direct numerical simulation of premixed turbulent combustion. Prog. Energy Combust. Sci. 12, 531-576.

Poinsot, T. \& Veynante, D. 2001 Theoretical and Numerical Combustion. Edwards.

Reveillon, J., Bray, K. \& Vervisch, L. 1998 Dns study of spray vaporization and turbulent micro-mixing. In AIAA 98-1028. 36th Aerospace Sciences Meeting and Exhibit, January 12-15, Reno NV.

Reveillon, J. \& VeRvisch, L. 2000 Accounting for spray vaporization in non-premixed turbulent combustion modeling: A single droplet model (sdm). Combustion and Flame 1 (121), 75-90.

ReVEillon, J. \& Vervisch, L. 2005 Analysis of weakly turbulent diluted-spray flames and spray combustion regimes. J. Fluid Mech. (537), 317-347.

Riley, J. \& G.S. PATterson, J. 1974 Diffusion experiments with numerically integrated isotropic turbulence. Phys. Fluids 17, 292-297.

Rogallo, R. S. 1981 Numerical experiments in homogeneous turbulence. Tech. Rep.. NASA, technical Memorandum No. 81315.

Samimy, M. \& Lele, S. 1991 Motion of particles with inertia in a compressible free shear layer. Phys. Fluids 8 (3), 1915-1923. 
Siggia, E. \& Patterson, G. 1978 Intermittency effects in a numerical simulation of stationary three-dimensional turbulence. J. Fluid Mech. 86, 567-592.

Simonin, O., Fevrier, P. \& Laviéville, J. 1993 On the spatial distribution of heavy-particle velocities in turbulent flow: from continuous field to particulate chaos. Journal of Turbulence 118, 97-118.

Sirovich, L., ed. 1991 New perspectives in Turbulence. Springer, Berlin.

Squires, K. D. \& Eaton, J. 1991 Preferential concentration of particles by turbulence. Phys. Fluids 3 (5), 1169-1178.

Vervisch, L. \& Poinsot, T. 1998 Direct numerical simulation of non-premixed turbulent flame. Annu. Rev. Fluid Mech. (30), 655-692.

WANG, L. \& MAXEY, M. 1993 Settling velocity and concentration distribution of heavy particles in homogeneous isotropic turbulence. J. Fluid Mech. 256, 27-68. 


\begin{tabular}{llll}
\hline & $S t=0.17$ & $S t=1.05$ & $S t=5.6$ \\
$\tau_{v}=\infty$ & $\mathrm{A}$ & $\mathrm{B}$ & $\mathrm{C}$ \\
$\tau_{v}=1 / 2 \tau_{0}$ & $\mathrm{~A}_{1}$ & $\mathrm{~B}_{1}$ & $\mathrm{C}_{1}$ \\
$\tau_{v}=1 \tau_{0}$ & $\mathrm{~A}_{2}$ & $\mathrm{~B}_{2}$ & $\mathrm{C}_{2}$ \\
$\tau_{v}=2 \tau_{0}$ & $\mathrm{~A}_{3}$ & $\mathrm{~B}_{3}$ & $\mathrm{C}_{3}$ \\
\hline
\end{tabular}

TABle 1. Configuration names, St : Stokes number, $\tau_{v}$ : evaporation delay. Non evaporating case : $\tau_{v}=\infty$ 


\begin{tabular}{|c|c|c|c|c|c|}
\hline & $\bar{Z}$ & $Z^{\prime}$ & $P(Z)$ & $E_{Z}$ & $E_{\xi}$ \\
\hline$(t \approx 0)$ & - & - & - & + & + \\
\hline$\left(0<t \leq \tau_{v}\right)$ & + & + & + & $+\rightarrow-(1)$ & - \\
\hline$\left(\tau_{v}<t\right)$ & - & + & $-(2)$ & - & + \\
\hline
\end{tabular}

TABLE 2. List of the effects due to preferential during the evaporation process. $t$ is the simulation time to be compared with the evaporation delay $\tau_{v} .(+)$ implies a major effect while $(-)$ indicates a minor impact. (1) turbulence effects, (2) classical Gaussian shape 


\section{Page 42 of 63}

42

J. Réveillon AND F.X. Demoulin

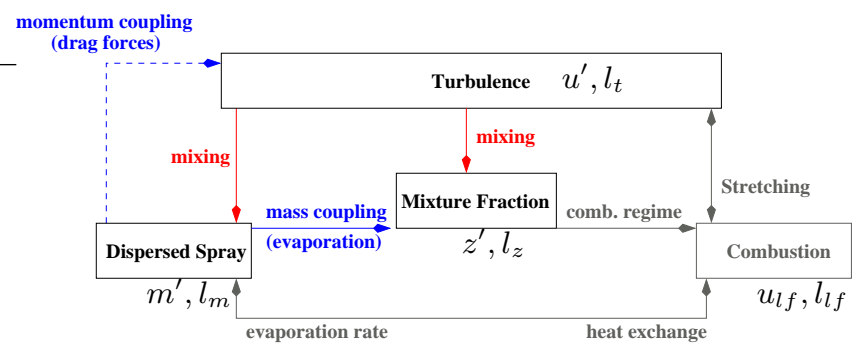

FIGURE 1. Sketch of direct and indirect mixing due to turbulence. General objective: analysis

of spray combustion. 


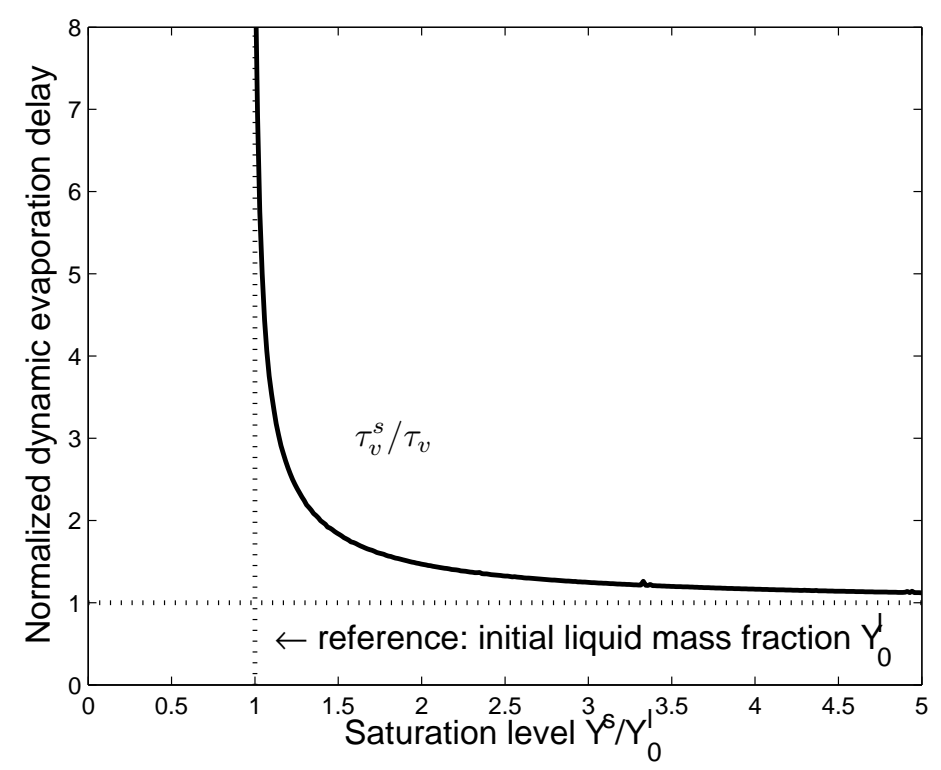

FIGURE 2. Evolution of the ratio $\tau_{v}^{s} / \tau_{v}$ versus the saturation value of the mixture fraction $Y^{s}$. The dynamic delay $\tau_{v}^{s}$ accounts for saturation phenomena while evaporation takes place. $\tau_{v}$ is the classical d-square law evaporation delay where the surrounding vapour is not taken into account. 


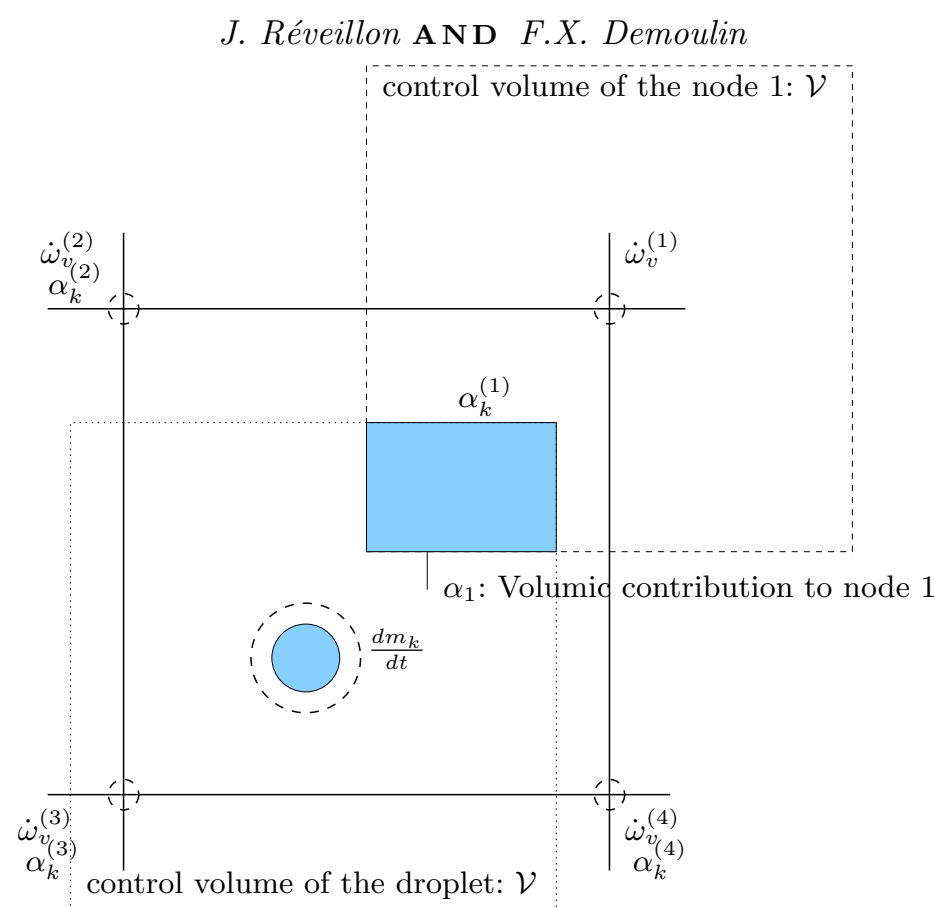

FiguRE 3. 2D Sketch of the repartition of droplet source terms on the closest Eulerian nodes.

A $3 \mathrm{D}$ repartition is done in the simulations. 


\section{Page 45 of 63}

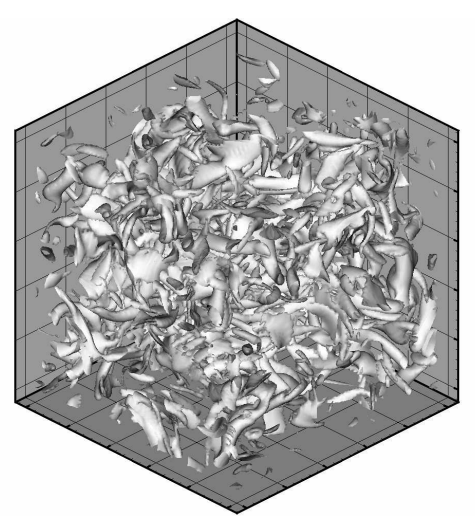

(a)

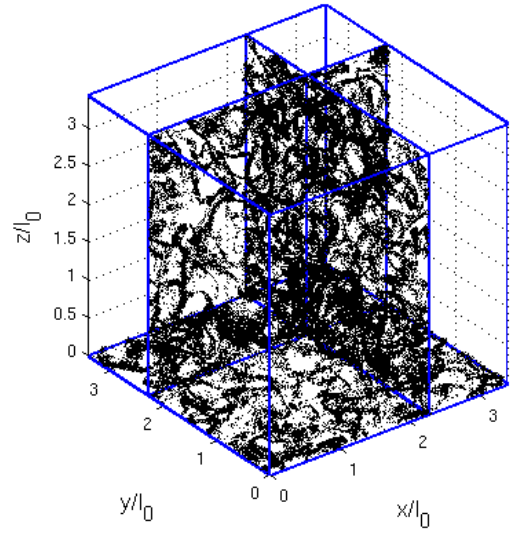

(b)

FiguRE 4. Examples of (a) vorticity contour and (b) spray dispersion 

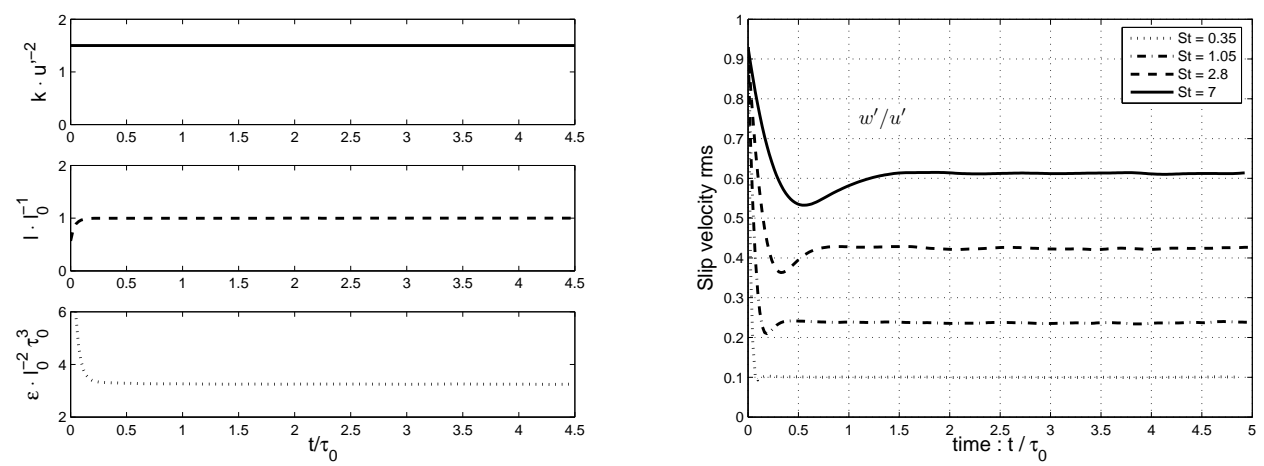

(a)

(b)
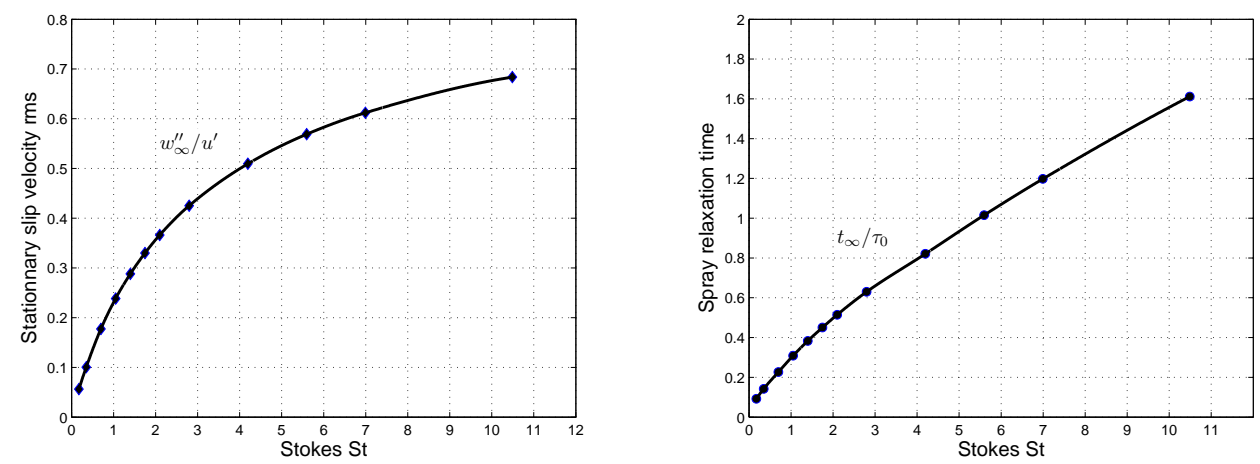

(c)

(d)

FIGURE 5. Statistically stationary convergence of turbulence and spray properties. (a): time evolution of the normalized (top) kinetic energy $k$, (middle) maximum energy length scale $l_{0}$, and (bottom) dissipation $\varepsilon$; (b): time evolution of particles slip velocity rms $\left(w^{\prime \prime}\right)$; (c): final $\left(t>t_{\infty}\right)$ stationary level of particles slip velocity $\operatorname{rms}\left(w_{\infty}^{\prime \prime} ;(\mathrm{d})\right.$ : spray relaxation time $\left(t_{\infty}\right)$ necessary for the spray to be in equilibrium with the turbulent carrier phase. 


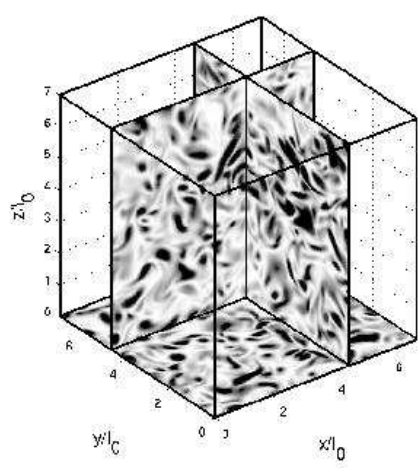

(a)

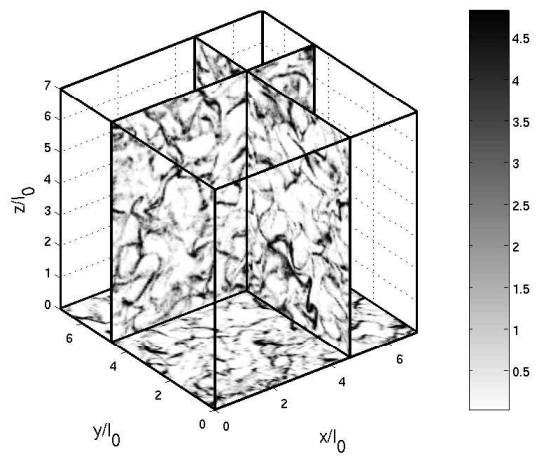

(c)

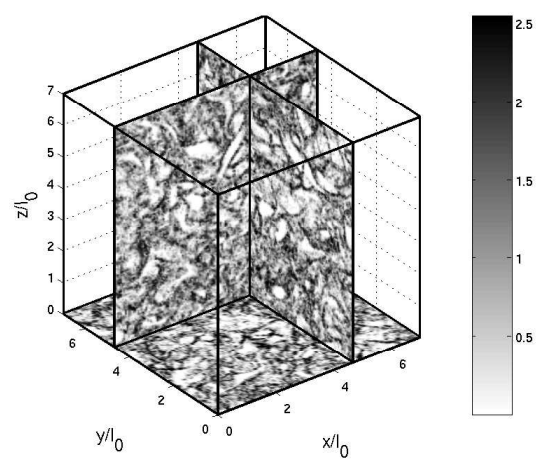

(b)

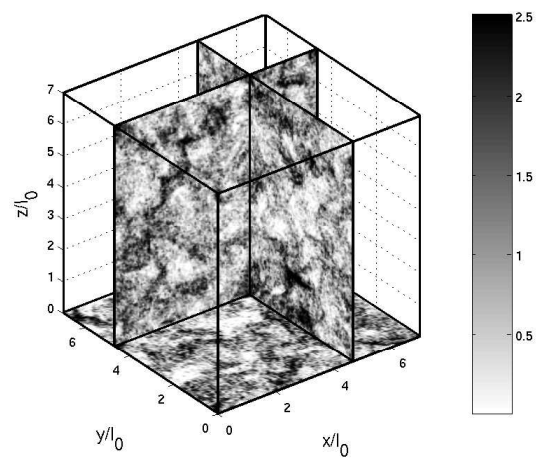

(d)

FiguRE 6. (a): Carrier phase vorticity $\tau_{0} \sqrt{\omega^{2}}$ and spray concentration areas $\xi / \bar{\xi}_{0}$, (b): $S t=0.17,(\mathrm{c}): S t=1.05,(\mathrm{~d}): S t=5.6$. 


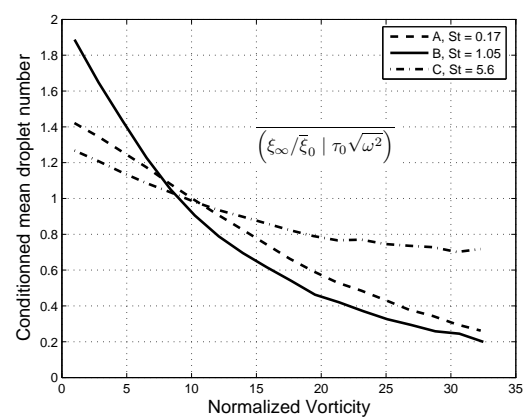

Figure 7 . Mean value of stationary droplet number $\xi_{\infty} / \xi_{0}$ conditioned by the carrier phase vorticity level $\omega$. 


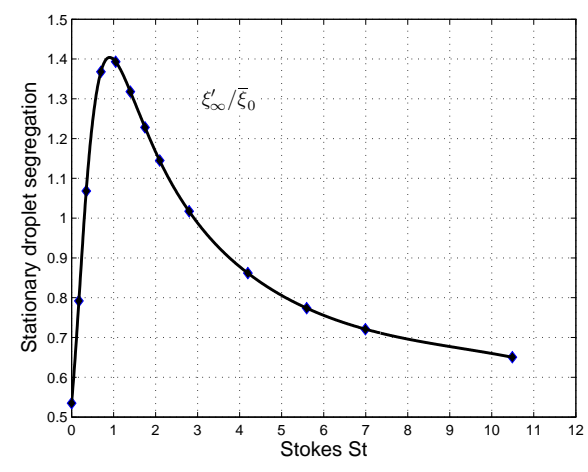

(a)

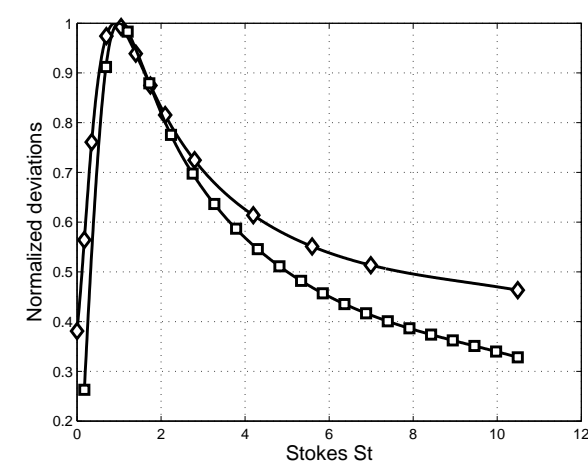

(b)

FIGURE 8. (a): Final stationary values of the droplet segregation parameter $\xi_{\infty}^{\prime}$ normalized by the initial mean value of $\xi: \bar{\xi}_{0}$. (b): Normalized standard deviations. Squares: Deviation from a Poisson distribution : $\Sigma$, diamonds: droplet segregation parameter $\xi_{\infty}^{\prime}$. 


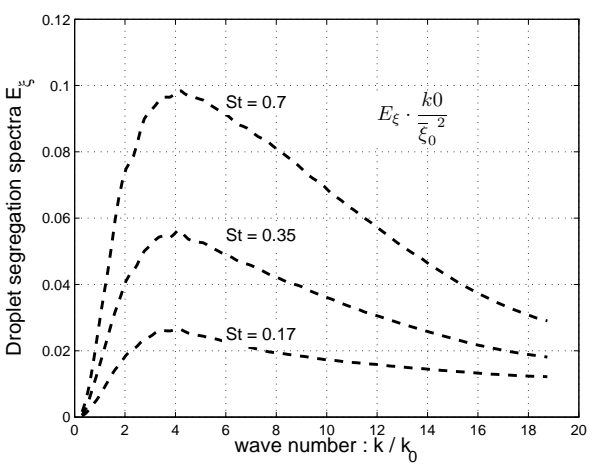

(a)

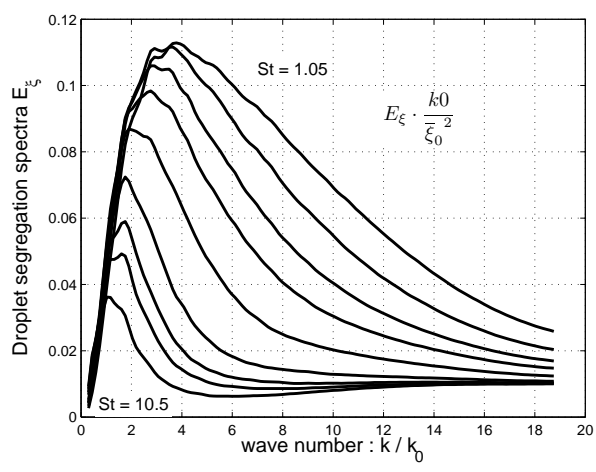

(b)

FIGURE 9. Stationary energy spectra of the droplet concentration parameter $\xi$ for various Stokes number. (a): small Stokes numbers $(S t<1)$; (b): large Stokes numbers $(S t>1)$. 


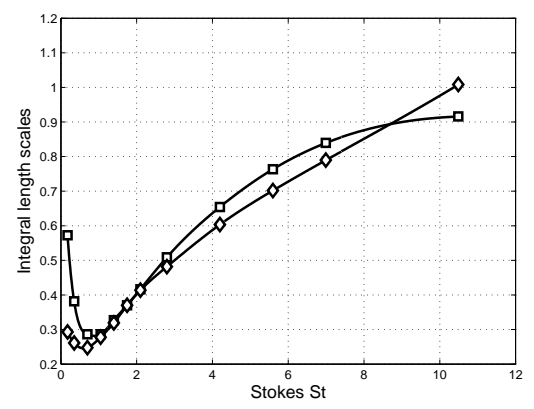

FiguRE 10. Characteristic cluster size determined with two different methods. Squares: cell size of the optimal deviation from the Poisson distribution $\Delta_{\Sigma m a x}$, diamonds: size of the most energetic clusters $l_{\xi}$ (determined from the energy spectrum of the droplet density). 


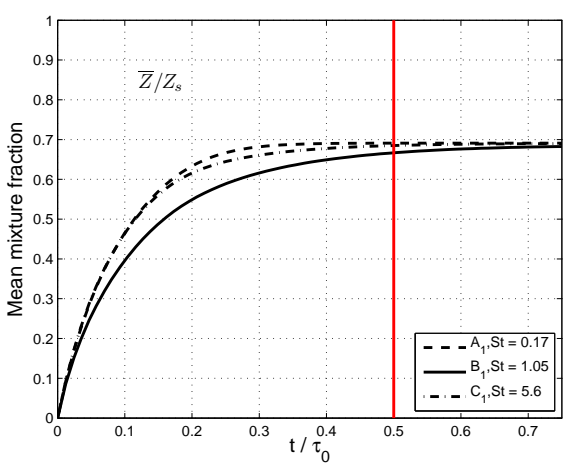

(a)

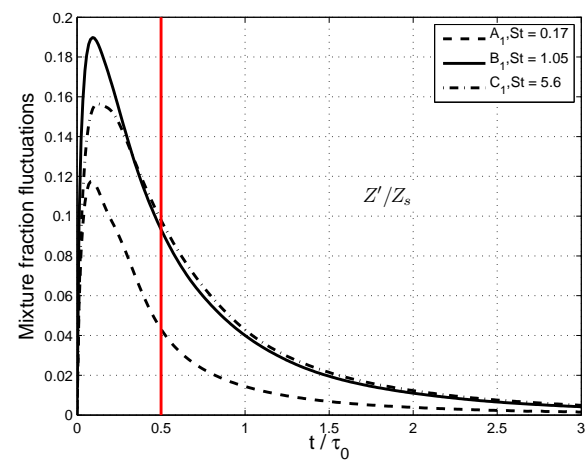

(b)

FigURE 11. Evolution of mixture fraction statistics in the domain, characteristic evaporation time $: 0.5 \tau_{0}$. Left : mean mixture fraction, right : deviation 


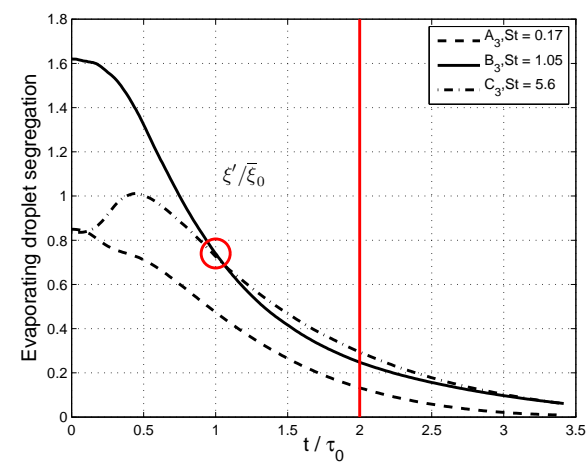

(a)

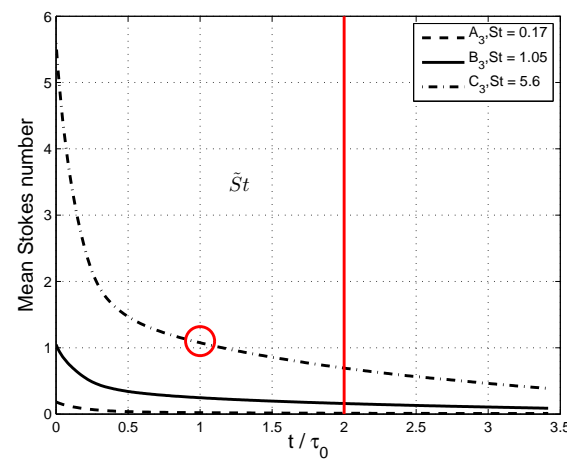

(b)

FIGURE 12. Droplet mean properties evolution, characteristic evaporation time : $2 \tau_{0}$. Left : droplet number fluctuations, right : mean Stokes number. 


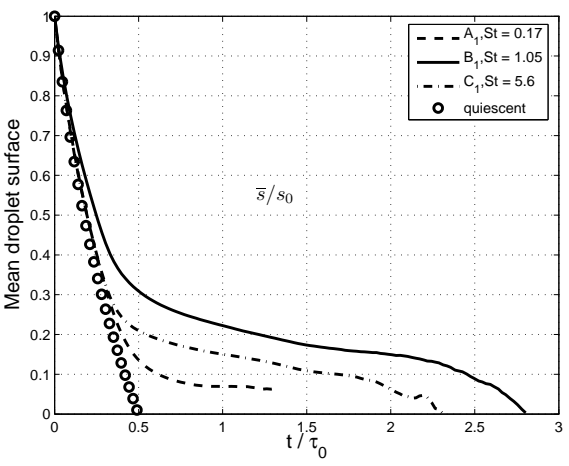

(a)

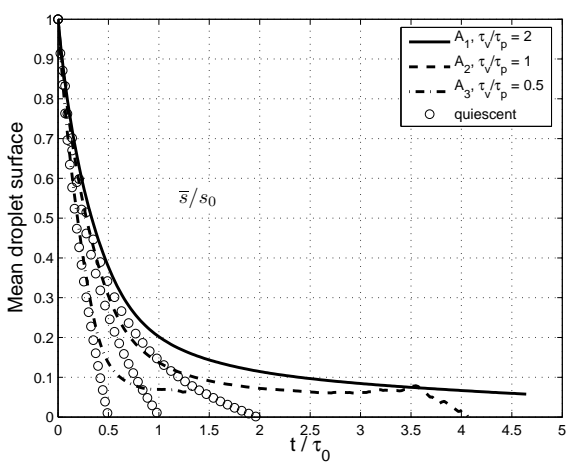

(b)

FIGURE 13. Droplet mean surface evolution. Symbols : droplet evaporation in a quiescent atmosphere. 
1)

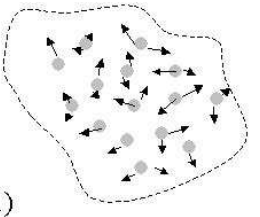

3a)

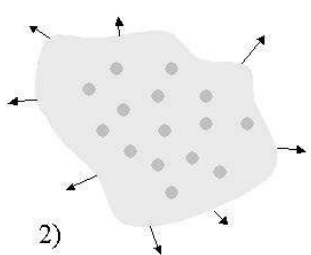

3b)

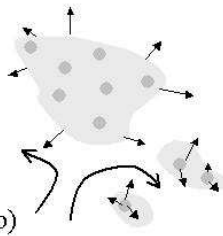

FIGURE 14. Sketch of the evaporation process 

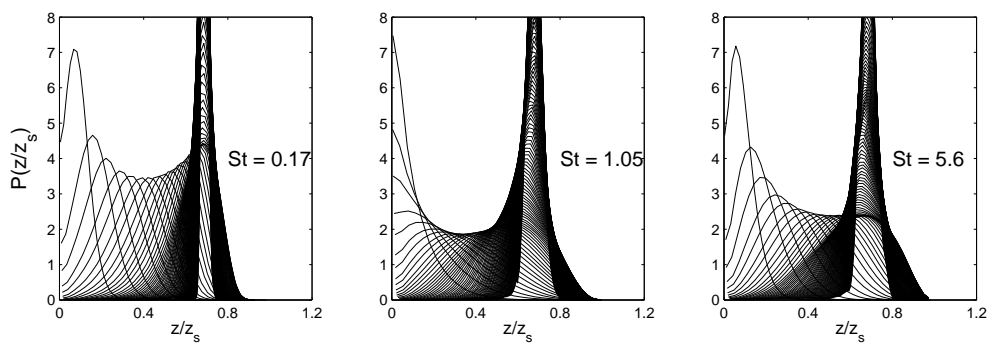

Figure 15. Time evolution of the PDF of $Z / Z_{s}$. $30 \mathrm{PDF}$ over one characteristic evaporation time. 

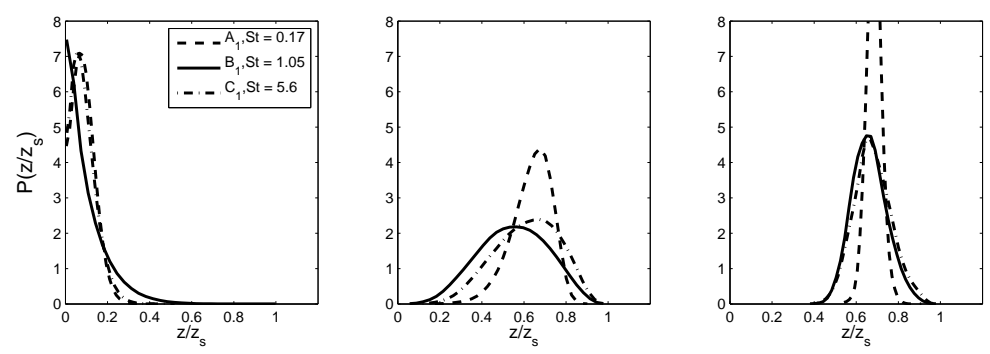

FiguRE 16. PDF of $Z$ for various Stokes number,left: $t=0.025 \tau_{0}$, centre: $t=0.5 \tau_{0}$ and

$$
t=1.15 \tau_{0}
$$




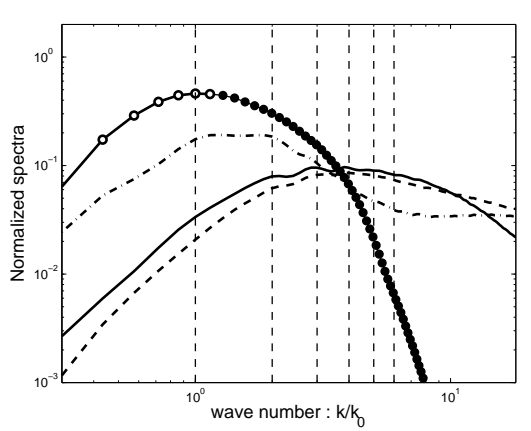

FIGURE 17. Variance normalized stationary spectra of $\xi$ fluctuations when non-evaporating spray is in equilibrium. Symbols: carrier phase kinetic energy spectrum (void circles: forced range, plain symbols: freely evolving range); dashed line: $A, S t=0.17$; solid line: $B, S t=1.05$; dot-dashed line: $C, S t=5.6$. 


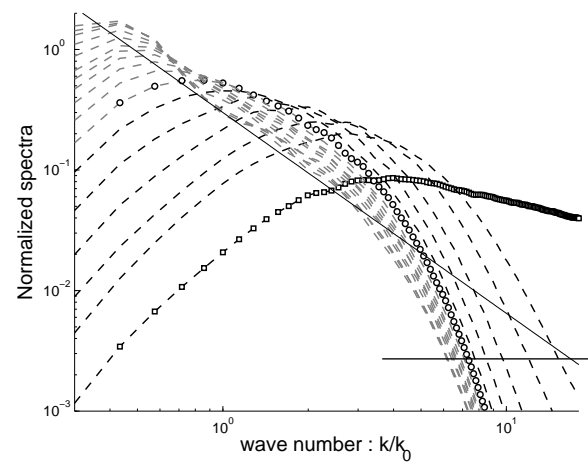

(a)

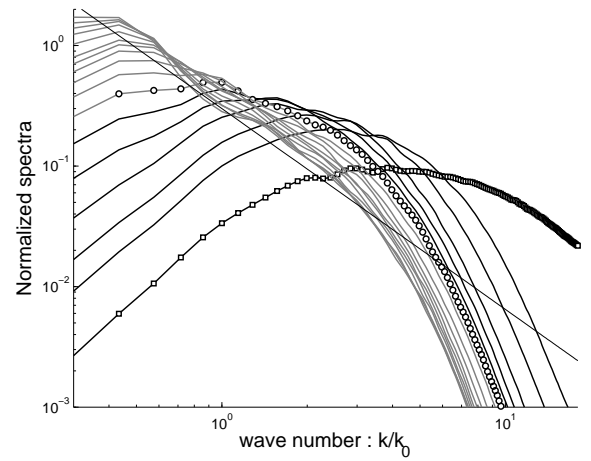

(c)

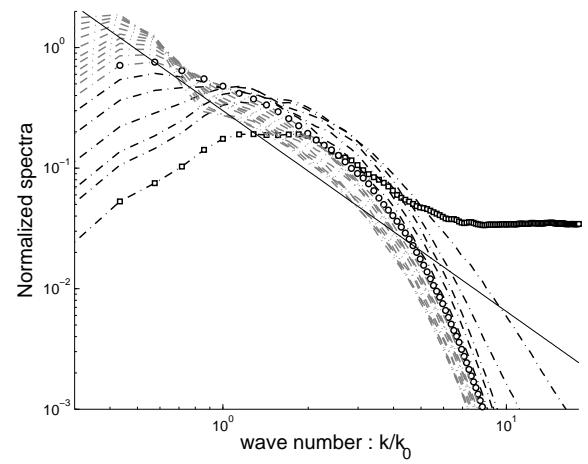

(e)

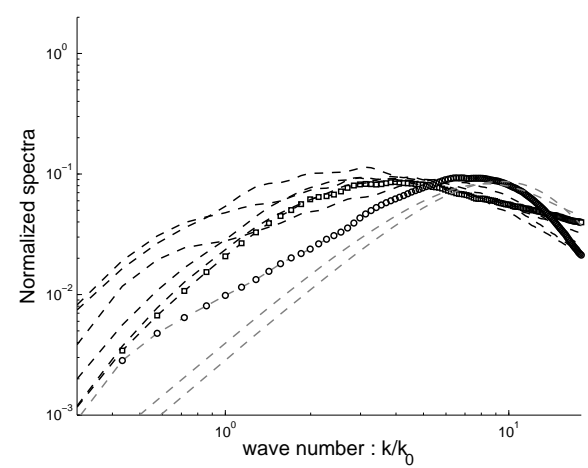

(b)

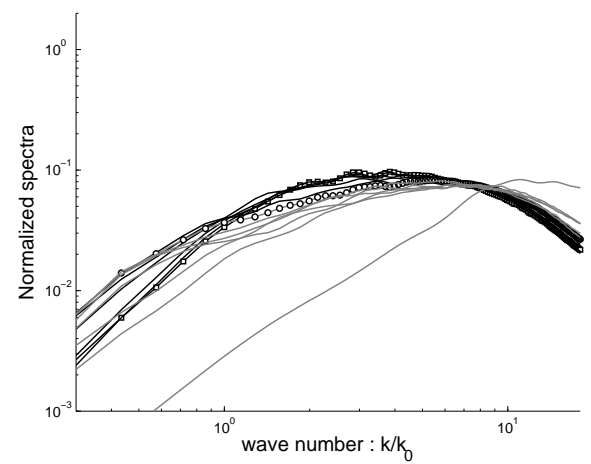

(d)

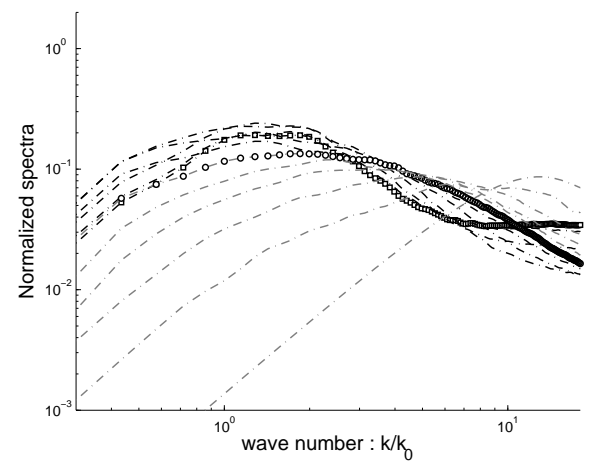

(f)

FiguRE 18. Time evolution of $E_{Z}$ (left) and $E_{\xi}$ (right) normalized spectra, $\tau_{v}=0.5 \tau_{0}$. Straight line: $k^{-5 / 3}$ range. Bold lines: evaporation starts, black lines: time $<\tau_{v}$, symbols: time $=\tau_{v}$, gray lines: time $\geq \tau_{v}$. (a): $E_{Z}, \quad S t=0.17,(\mathrm{~b}): E_{\xi}, \quad S t=0.17,(\mathrm{c}): E_{Z} \quad, \quad S t=1.05$, (d): $E_{\xi}, S t=1.05,(\mathrm{e}): E_{Z}, S t=5.6,(\mathrm{f}): E_{\xi}, S t=5.6$. 


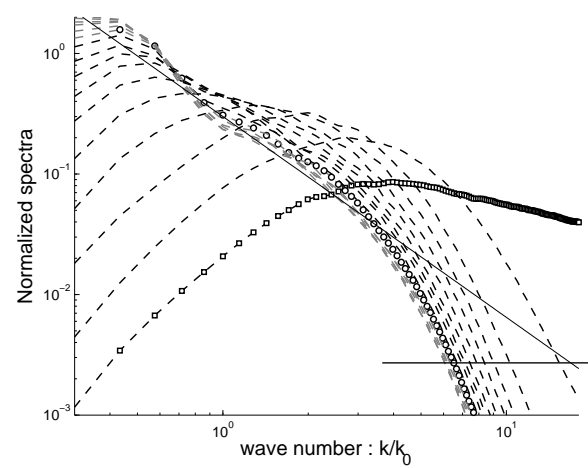

(a)

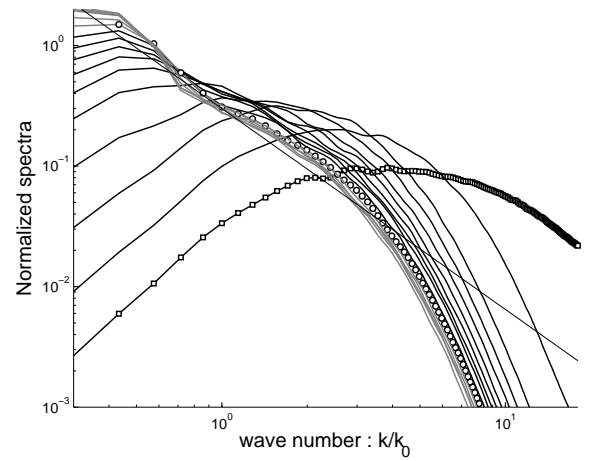

(c)

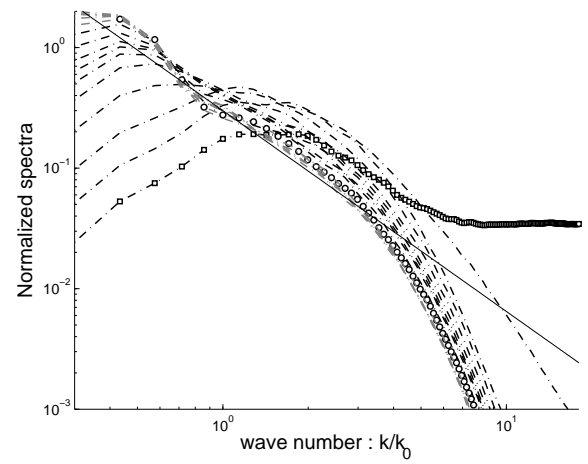

(e)

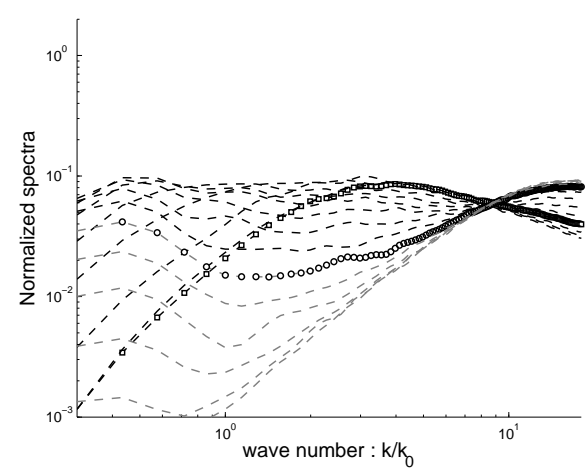

(b)

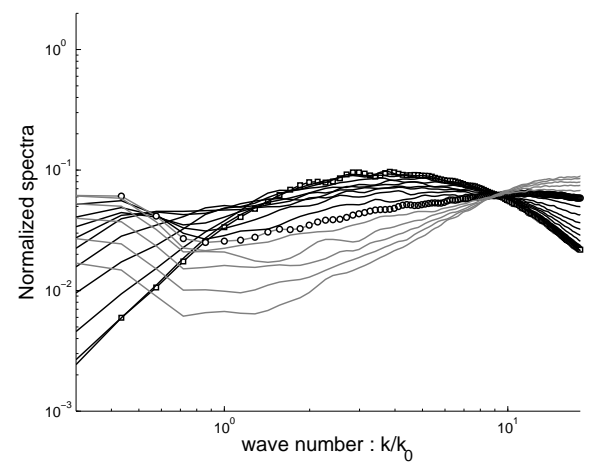

(d)

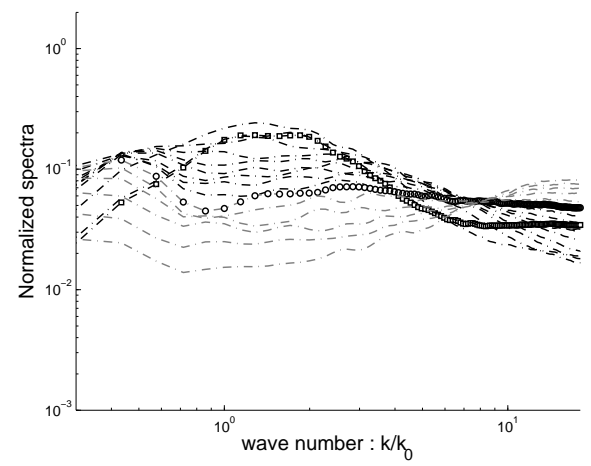

(f)

FigurE 19. Time evolution of $E_{Z}$ (left) and $E_{\xi}$ (right) normalized spectra, $\tau_{v}=2 \tau_{0}$. Straight line: $k^{-5 / 3}$ range. Bold lines: evaporation starts, black lines: time $<\tau_{v}$, symbols: time $=\tau_{v}$, gray lines: time $\geq \tau_{v}$. (a): $E_{Z} \quad, \quad S t=0.17,(\mathrm{~b}): E_{\xi} \quad, \quad S t=0.17,(\mathrm{c}): E_{Z} \quad, \quad S t=1.05,(\mathrm{~d})$ : $E_{\xi} \quad, \quad S t=1.05,(\mathrm{e}): E_{Z} \quad, \quad S t=5.6,(\mathrm{f}): E_{\xi} \quad, \quad S t=5.6$. 


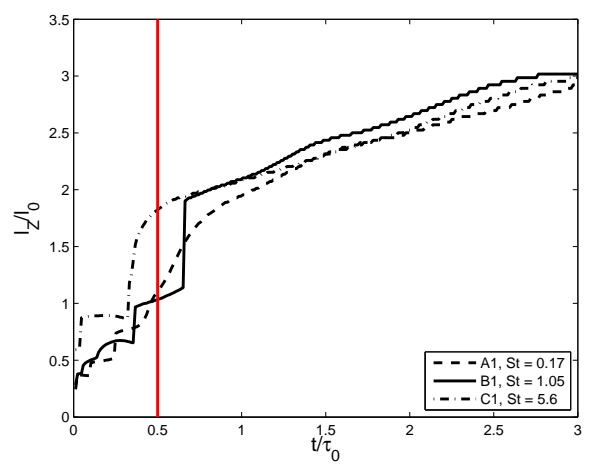

(a)

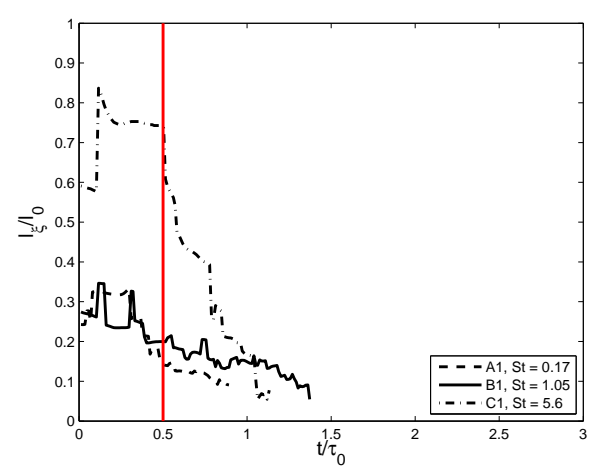

(b)

Figure 20. Time evolution of the length scales $l_{Z}$ (a) and $l_{\xi}$ (b), when $\tau_{v}=0.5 \tau_{0}$, corresponding to the size of the most energetic structures of the turbulent fields $Z$ and $\xi$, respectively. 


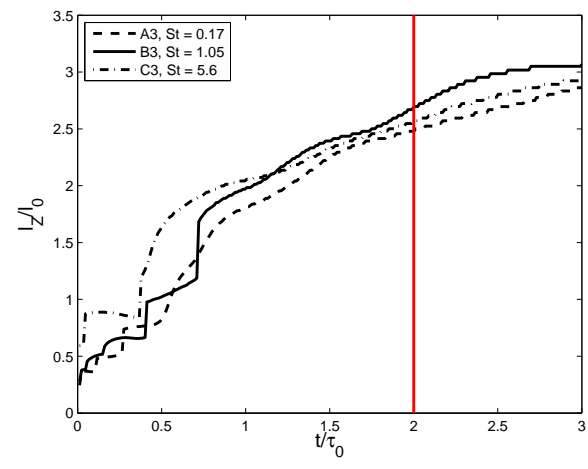

(a)

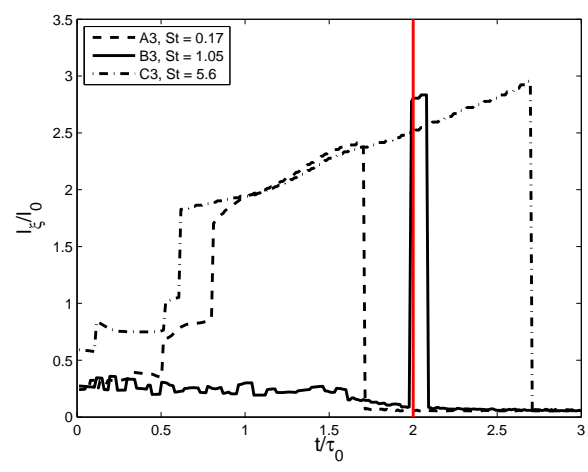

(b)

Figure 21. Time evolution of the length scales $l_{Z}$ (a) and $l_{\xi}$ (b), when $\tau_{v}=2 \tau_{0}$, corresponding to the size of the most energetic structures of the turbulent fields $Z$ and $\xi$, respectively. 


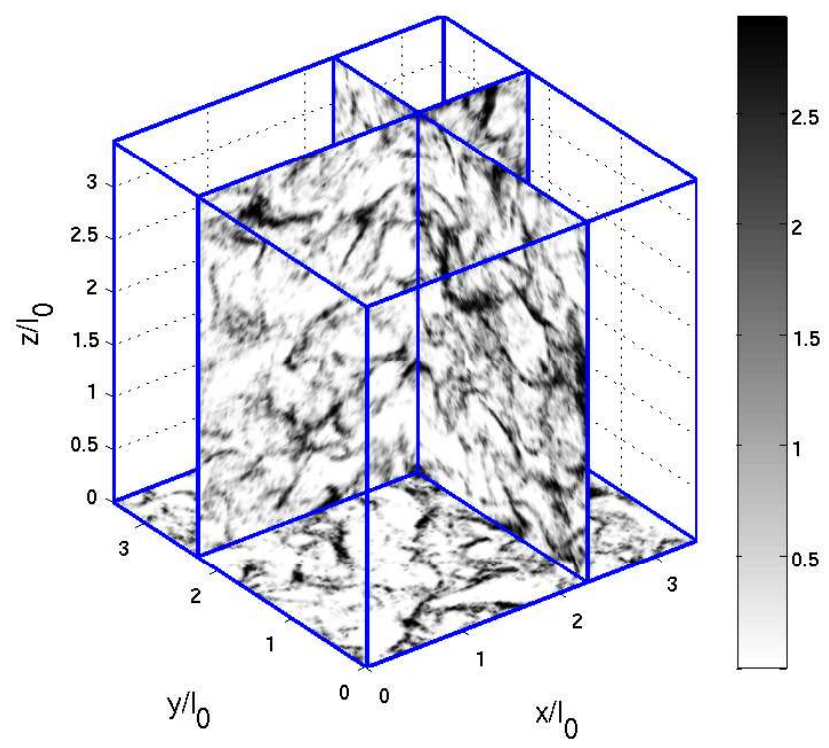

(a)

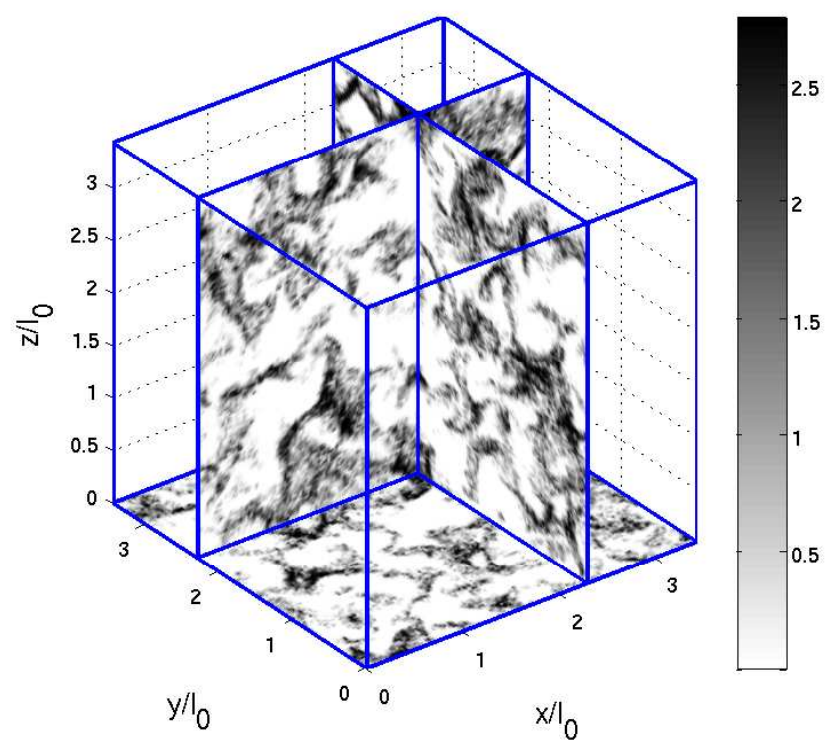

(b)

Figure 22. Spray density field when $t=\tau_{v}$ and $S t=5.6$. The remaining droplets are embedded in vapour pockets. (a) $\tau_{v}=2 \tau_{0}$, (b) $\tau_{v}=\tau_{0} / 2$ 